\title{
Digital image-based modeling applied to the homogenization analysis of composite materials
}

\author{
K. Terada, T. Miura, N. Kikuchi
}

\begin{abstract}
Absract The systematic methodologies to derive accurate microstructural models are developed for studying the mechanical behaviors of composite materials. Since the geometric information of a microstructure is often given by an image or a set of images, the direct interpretation of the geometry is possibly by digitizing it. By identifying each pixel or voxel with a finite element (FE) and accompanying appropriate image processing, an FE model can be automatically generated. It is also emphasized that the digitized models can be suitable for solving the FE equations by utilizing the uniformity of the FE mesh. The finite element analysis (FEA) with the homogenization method enables the prediction the thermo-mechanical behavior of the periodic microstructure (unit cell) as well as the global mechanical response of a structural component, while we are taking into account the specific effect of the geometric structural configuration of the microstructure through digitization. Several kinds of the digitizing techniques are presented to illustrate the potential of digital image-based (DIB) FE modeling of the unit cell. Keeping the microstructural design in mind, the modification of the plane image is introduced and the virtual realization of the unit cell geometry is presented so that a microstructural analysis utilizing the homogenization method would be realistic.
\end{abstract}

Communicated by S. N. Atluri, 19 February 1997

K. Terada

Research Associate, Structural System Engineering Laboratory, Department of Naval Architecture and Ocean Engineering, The University of Tokyo,

7-3-1 Hongo, Bunkyo-ku, Tokyo 113, Japan

T. Miura, Graduate Student

Department of Aerospace Engineering,

The University of Michigan,

Ann Arbor, Michigan 48109, USA

N. Kikuchi, Professor

Computational Mechanics Laboratory

Department of Mechanical Engineering and Applied Mechanics, The University of Michigan,

2125 EECS, Ann Arbor, Michigan 48109-2215, USA

Correspondence to: $\mathrm{K}$. Terada

The authors are supported by NSF MSS-93-01807, US Army TACOM, DAAE07-93-C-R125 and US Navy ONR, N00014-94-10022 and AFOSR-URI program, DoD-G-F49620-93-0289.
1

\section{Introduction}

The mathematical theory of homogenization has its basis on the method of two-scale asymptotic expansion and has been applied to various kind of systems of governing equations for composite materials which are assumed to be formed by spatial repetition of unit cells, see, e.g., Sanchez-Palencia (1980). The asymptotic homogenization method provides both micro and macroscopic boundary value problems so that the effective properties can be derived and the micro-mechanical behaviors can be evaluated from an overall structural response; these processes are called homogenization and localization, respectively. The global-local nature of the method was utilized to successfully analyze the mechanical behavior of a composite material in linear elasticity together with the finite element method (FEM) (Lene (1982), Guedes and Kikuchi (1991)) and extended to three-dimensional elasto-plastic problems by Terada and Kikuchi (1995). However, the things to be emphasized are not only such a global-local nature but also its capability of handling almost any kind of heterogeneities. That is, its continuum-based formulation does not impose any restriction on the geometric configuration of the microstructure as far as the locally periodic distribution of unit cells is assumed. Therefore, it is possible to study the mechanical behavior of a complex microstructure by the FEM if an FE mesh can be generated properly so that the homogenization with FEM can capture the effects of the original geometric configuration.

However, it is not straightforward to make a FE mesh of a unit cell even if the complete geometry is identifiable and if an automatic mesh generator or/and solid modeler is available. In fact, it is not always the case the microstructures have simple geometry and, in some cases, the microstructural geometry may be too complex to be identified. Although conventional FE modeling inevitably idealizes the geometry, there may not be sufficient amount of geometric information in the idealized model and, especially for naturally formed composites such as geo-

materials or bio-tissues. Such idealization itself seems not to make sense because the irregular configuration features such materials. Therefore, we need to develop a systematic way to construct an accurate microstructural model so that the homogenization results can reflect the actual geometry of the microstructure.

Several approaches to deal with accurate geometry modeling exist in the literature. For example, Ghosh and Moorthy (1995) modeled the microstructure using a network of multi-sided convex Voronoi polygons using an 
automated image analysis system for micrographs. By combining the Voronoi cell finite element method with the asymptotic homogenization method, they made it possible to qualitatively characterize a random feature of the heterogeneities of a certain kind of metal matrix composites. Yet it seems that both the modeling and formulation are somewhat complicated and may have difficulties when concerning natural composite materials such as human bone. Another approach introduced by Berryman (1987) combined digital image processing and statistical data handling. He utilized $n$-point correlation functions from digitized binary data of an actual specimen to characterized the geometry and then estimated parameters for a certain empirical relationship of derive a Darcy's constant of bedrock. Although the statistical argument and the empirical relation to estimate effective moduli are used instead of the homogenization method, its geometrical representation by digital images is direct and automated. Noticing the potential of the digital images, Hollister and Kikuchi (1994) also utilized the digital imaging technique to recognize the microstructural geometry in order to analyze the bone tissue by the asymptotic homogenization method with FEM. Complete three-dimensional images of an actual bone microstructure were obtained from microCT scanner. After an appropriate thresholding, they identified each volume element (voxel) with a finite element to make a stress/deformation analysis of a bone structure. Although they reported some disadvantages, their approach, namely the digital image-based (DIB) modeling with the homogenization method, seems to be unique and indicates the possibility of various kinds of applications in computational mechanics. In this context, some of the advantages and the applications should be studied for two-dimensionally captured images since most of the microstructures have such a fine scale that we cannot capture it utilizing micro-CT scanner. Especially for industrial (or manufactured) composite materials, the geometric information of the microstructure is given only by a micrograph in most cases.

In this paper, we will present several types of geometric modeling techniques based on digital images in conjunction with RVE analysis by the asymptotic homogenization method. First, we will briefly review the theory of homogenization method for linear thermo-elasticity. Second, the digital image processing techniques are described based on the plane image of a metal matrix composite (MMC), aiming at the DIB-FE modeling which has not been studied very throughly. Third, the FE procedure based on the digitized and processed images is summarized, focussing on actual data handling, and then the feasibility of the FE approximation is examined by an illustrative numerical example. Fourth, we will verify the validity of RVE homogenization analysis using micrographs of MMC with the systematic DIB modeling. In the last section, we will present two examples of 3D DIB-FE modeling when the available information is one captured plane image by introducing several image manipulations according to requests. One of the examples is the analysis of thermal stress, in which we illustrate how to change the volume fraction of constituents by image manipulation and will be found to be related to microstructural design of a composite. The other example concerns the method of estimating the 3D configuration of the microstructure, which is used in 3D stress analysis, from limited geometry information. In order to achieve such a virtual geometry, we propose a sort of triangulation technique for two dimensional images. The novel geometry and FE modeling method in this paper could be helpful for analysis, design, manufacturing and others. Thus, our objective is to show the potential of digital images by presenting some of the applications and to provide insight into their use in computational engineering.

\section{2}

\section{The asymptotic homogenization method}

Let us consider a general composite structure which is composed of several different materials. The heterogeneous mechanical characteristics of the structure come from its microstructures, which are assumed to be linearly elastic solids. We assume that an microstructure can be chosen to be a representative volume element (RVE) and that it is spatially distributed in locally periodic manner. This microstructure is usually called a unit cell since it can reproduce a local region by repeating the unit. We recall that the homogenization theory for linear elasticity considers such a composite structure with a unit cell of a characteristic length $\varepsilon$ which relates a microscopic scale $y$ and macroscopic one $\boldsymbol{x}$ such that $\boldsymbol{y}=\boldsymbol{x} / \varepsilon$. Indicating the dependency of the deformation on the heterogeneity of the microstructure by the superscript $\varepsilon$, we relate the Cauchy stress, $\sigma_{i j}^{\varepsilon}(\boldsymbol{x})$, to an infinitesimal strain, $e_{k h}\left(\mathcal{u}^{\varepsilon}(\boldsymbol{x})\right)$, and a thermal strain by the following relation:

$\sigma_{i j}^{\varepsilon}=a_{i j k h}^{\varepsilon}\left(e_{k h}\left(\boldsymbol{u}^{\varepsilon}\right)-\Delta T \alpha_{k h}^{\varepsilon}\right)=a_{i j k h}^{\varepsilon} e_{k h}-\Delta T \beta_{i j}^{\varepsilon}$

where $\boldsymbol{u}^{\varepsilon}$ is the displacement, $a_{i j k h}^{\varepsilon}(\boldsymbol{x} / \varepsilon)=a_{i j k h}(\boldsymbol{y})$ is the elasticity tensor, $\Delta T$ is the temperature change, and $\alpha_{k h}^{\varepsilon}(\boldsymbol{x})$ is the thermal expansion coefficient (CTE) tensor. Here $e_{k h}$ and $\beta_{i j}^{\varepsilon}(\boldsymbol{x})$ are respectively defined as

$e_{k h}\left(\boldsymbol{u}^{\varepsilon}\right)=\frac{1}{2}\left(\frac{\partial u_{k}^{\varepsilon}}{\partial x_{h}}+\frac{\partial u_{h}^{\varepsilon}}{\partial x_{k}}\right)$

and

$\beta_{i j}^{\varepsilon}=a_{i j k h}^{\varepsilon} \alpha_{k h}^{\varepsilon}$

Then, the variational formulation of a boundary value problem for the structure is given by:

$$
\begin{aligned}
\int_{\Omega^{\varepsilon}} a_{i j k h}^{\varepsilon}(\boldsymbol{x}) \frac{\partial v_{i}^{\varepsilon}}{\partial x_{j}} \frac{\partial u_{k}^{\varepsilon}}{\partial x_{h}} \mathrm{~d} x= & \int_{\Omega^{\varepsilon}}\left\{\Delta T \beta_{i j}^{\varepsilon} \frac{\partial v_{i}^{\varepsilon}}{\partial x_{j}}+b_{i}^{\varepsilon} v_{i}^{\varepsilon}\right\} \mathrm{d} x \\
& +\int_{\Gamma_{l}} \hat{t}_{i}(\boldsymbol{x}) v_{i}^{\varepsilon} \mathrm{d} x \quad \forall \boldsymbol{v}^{\varepsilon}
\end{aligned}
$$

where $\hat{t}_{i}$ is the traction vector, $b_{i}^{\varepsilon}$ is the body force vector and $v_{i}^{\varepsilon}$ is the virtual displacement field. Following the method of two-scale asymptotic expansion, we expand the displacement and virtual fields as follows:

$$
\left\{\begin{array}{l}
\boldsymbol{u}^{\varepsilon}=\boldsymbol{u}^{0}(\boldsymbol{x}, \boldsymbol{y})+\varepsilon \boldsymbol{u}^{1}(\boldsymbol{x}, \boldsymbol{y})+\varepsilon^{2} \boldsymbol{u}^{2}(\boldsymbol{x}, \boldsymbol{y})+\cdots \\
\boldsymbol{v}^{\varepsilon}=\boldsymbol{v}^{0}(\boldsymbol{x}, \boldsymbol{y})+\varepsilon \boldsymbol{v}^{1}(\boldsymbol{x}, \boldsymbol{y})+\varepsilon^{2} v^{2}(\boldsymbol{x}, \boldsymbol{y})+\cdots
\end{array}\right.
$$


Then the theory assures that, as $\varepsilon$ goes to zero, the displacement field, $\boldsymbol{u}^{\varepsilon}$, tends to the average displacement field, $\boldsymbol{u}^{0}$, which is independent of $\boldsymbol{y}$. That is, if the domain were occupied by some homogenized medium, then $\boldsymbol{u}^{0}$ satisfy the following macroscopic problem:

$$
\begin{aligned}
\int_{\Omega} a_{i j k h}^{H} \frac{\partial u_{k}^{0}}{\partial x_{h}} \frac{\partial v_{i}^{0}}{\partial x_{j}} \mathrm{~d} x= & \int_{\Omega} \Delta T a_{i j k h}^{H} \alpha_{k h}^{H} \frac{\partial v_{i}^{0}}{\partial x_{j}} \mathrm{~d} x \\
& +\int_{\Omega} b_{i}^{H} v_{i}^{0} \mathrm{~d} x+\int_{\Gamma_{l}} \hat{t}_{i} v_{i}^{0} \mathrm{~d} \Gamma \quad \forall \boldsymbol{v}^{0}
\end{aligned}
$$

where $a_{i j k h}^{H}$ is the homogenized elasticity tensor, $\alpha_{k h}^{H}$ is the homogenized thermal expansion coefficient tensor, $b_{i}^{H}$ is the homogenized body force vector. These homogenized values are calculated using the characteristic deformations $\chi_{i}^{k h}(\boldsymbol{y})$ and $\psi(\boldsymbol{y})$, which are the solutions of the following governing equation, respectively:

$\int_{Y} a_{i j l m} \frac{\partial w_{i}}{\partial y_{j}} \frac{\partial \chi_{l}^{k h}}{\partial y_{m}} \mathrm{~d} y=\int_{Y} a_{i j k h} \frac{\partial w_{i}}{\partial y_{j}} \mathrm{~d} y, \quad \forall \boldsymbol{w}$,

and

$\int_{Y} a_{i j l m} \frac{\partial w_{i}}{\partial y_{j}} \frac{\partial \psi_{l}}{\partial y_{m}} \mathrm{~d} y=\int_{Y} \beta_{i j} \frac{\partial w_{i}}{\partial y_{j}} \mathrm{~d} y, \quad \forall \boldsymbol{w}$

where the characteristic deformations and the virtual displacement $w_{i}$ are defined within a unit cell region $Y$. After solving these equations subjected to the periodic boundary conditions, the homogenized values are computed by the formulae

$a_{i j k h}^{H}=\frac{1}{Y} \int_{Y}\left(a_{i j k h}-a_{i j l m} \frac{\partial \chi_{l}^{k h}}{\partial y_{m}}\right) \mathrm{d} y$,

$\alpha_{l m}^{H}=c_{l m i j}^{H} \frac{1}{Y} \int_{Y}\left(a_{i j k h} \alpha_{k h}-a_{i j k h} \frac{\partial \psi_{k}}{\partial y_{h}}\right) \mathrm{d} y$,

and

$b_{i}^{H}=\frac{1}{Y} \int_{Y} b_{i} \mathrm{~d} y$.

Here $|Y|$ indicates the volume of the unit cell and $c_{l m i j}^{H}$ is the compliance tensor defined by the inverse of the elasticity tensor, i.e., $c_{l m i j}^{H}=\left(a_{l m i j}^{H}\right)^{-1}$.

The microscopic deformation is recognized as the first-order term of the expanded displacement by the following relation:

$u_{i}^{1}(\boldsymbol{x}, \boldsymbol{y})=w_{i}^{1}(\boldsymbol{x}, \boldsymbol{y})+\tilde{u}_{i}^{1}(\boldsymbol{x})$

where $\tilde{u}_{i}^{1}(\boldsymbol{x})$ is a constant within a unit cell and the $w_{i}^{1}(\boldsymbol{x}, \boldsymbol{y})$ represents the deformation of the unit cell and is defined as

$w_{i}^{1}(\boldsymbol{x}, \boldsymbol{y})=-\chi_{i}^{k h}(\boldsymbol{y}) \frac{\partial u_{k}^{0}(\boldsymbol{x})}{\partial x_{h}}+\Delta T(\boldsymbol{x}) \psi_{i}(\boldsymbol{y})$.

Therefore, once the macroscope problem (6) is solved for the average displacement, $u_{k}^{0}(\boldsymbol{x})$ and $\Delta T(\boldsymbol{x})$ is given, these values are localized to give the micromechanical response of the unit cell. Since the asymptotic expansion of Cauchy stress deduced from (1) as $\sigma_{i j}^{\varepsilon}=\sigma_{i j}^{0}(\boldsymbol{x}, \boldsymbol{y})+\varepsilon \sigma_{i j}^{1}(\boldsymbol{x}, \boldsymbol{y})+\cdots$,

the microscopic stress is defined by

$$
\begin{aligned}
\sigma_{i j}^{0} & =a_{i j l m}\left[e_{l m}^{0}\left(\boldsymbol{w}^{1}\right)-\Delta T \alpha_{i j}\right] \\
& =\left(a_{i j k h}-a_{i j l m} \frac{\partial \chi_{l}^{k h}}{\partial y_{m}}\right) \frac{\partial u_{k}^{0}}{\partial x_{h}}-\Delta T \beta_{i j}
\end{aligned}
$$

while $e_{l m}^{0}\left(\boldsymbol{w}^{1}\right)$ is the microscopic strain.

We have skipped the several steps in the formulation and omitted some explanations and will not be involved much in quantitative discussion of homogenization results, since they are not relevant to our present interest. One can refer to literature, for example, Guedes and Kikuchi (1991) or Duvaut (1983) for the detailed derivation of the homogenization formulae. Instead, our focus in this paper is to illustrate FE modeling techniques for the unit cell by using systematic operations of digital images. In the next section, the utilization of the digital images in the FE modeling will be described.

\section{3}

\section{Digital imaging of microstructure}

\section{1}

\section{Introductory remarks}

"An image is a spatial representation of an object.", Haralick and Shapiro (1994). Necessary information in mechanics is usually given by an image or a set of several images that is obtained in a measurement or experimental procedure. The information is eventually converted into a screen image on a cathode ray tube (CRT) of a digital computer. Now the idea is that we directly convert the bitmap information on the computer into a geometry model for a numerical analysis such as Finite Element Analysis (FEA). This digital image-based (DIB) FEM was originated by Hollister and Kikuchi (1994) for studying a bone microstructure by the homogenization method. Although we basically follow their procedure, we shall focus our attention on data structure and its handling operations on computers.

The method presented here utilizes both hardware and software capability available. Indeed, most of the processes in digital image processing have been performed using commercial software on personal computers; for example, Adobe Photoshop ${ }^{\mathrm{TM}}$ of (CAdobe Systems, Incorporated or by NIH Image (PDS) of National Institute of Health on the C Macintosh series of $\odot$ Apple Computer, Inc. or on $\odot$ IBM PC series with MS-Windows ${ }^{\text {TM }}$ of $\bigodot$ Microsoft Corporation. Although there are many operations in image processing such as filtering, blurring, distorting, and etc. (Dougherty 1994), we need only a few of them for the present purpose, i.e., the computational or FE modeling. For FEA, we have developed computer programs for the homogenization analyses by FORTRAN language on UNIX Engineering Workstations (EWS). Also, the FORTRAN language is utilized to manipulate the data for other purposes, which will be introduced in the applications in the DIB modeling.

The whole procedure can be divided into major four parts. The first step consists of capture and sampling, 
which are usually assumed to have been done. Selecting and thresholding, which are probably the most important operations, in the second step are the operations directly related to the FEM in the homogenization analysis. The third step contains exporting and, it necessary, adjusting. While the first three are involved in the pre-processing of FEA, the last process includes both the main part and postprocessing of the FEA of the homogenization method, details of which will be discussed in the next section. Prior to or during the FEA, the stacking may be processed using the exported data to construct the three-dimensional structure. Focusing the attention on the second and the third operations and the stacking technique, we describe each process below in order.

\section{2}

\section{Capture}

At the very beginning, prior to digitization, an image must be captured by a sensor which is chosen dependent on the desired formation modality. Typical sensors may be optical devices such as scanning electron microscopes (SEM) or micro-CT scanners. Using those devices, the image formation process must be carefully done by an operator so that the necessary information would be contained in the image. Since the details seem to be beyond the extent of our present discussion, let us assume that we have obtained appropriate images of the microstructure of a composite; there is little noise and distinctive phases can be identified in the image. Even if noise was present, it can be eliminated by thresholding later. Figure 1 shows a micrograph of a metal matrix composite (MMC) composed of NiAl-matrix and $\mathrm{Cr}$-fiber from SEM which is obtained in this capture process.

\section{3}

\section{Sampling (digitization and quantization)}

Once an image has been captured, the next stage is the sampling which is a process where the image is converted into numerical form in digital computers. The sampling consists of two sequential processes; (spatial) digitization and quantization. The digitization is accomplished by partitioning the area of the image into a finite twodimensional array composed of small uniform cells (res-

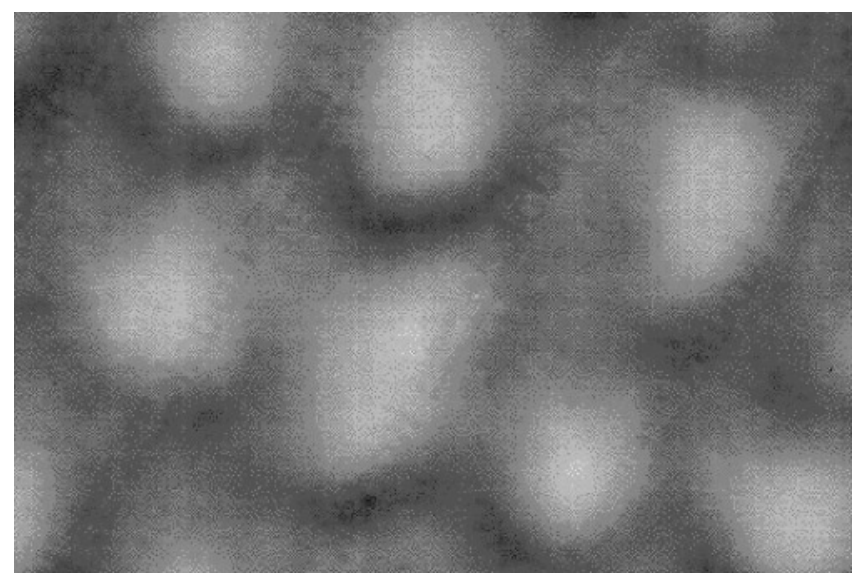

Fig. 1. Micrograph of MMC by SEM (Captured Image) olution cells). In the quantization process, a representative image value is assigned to each cell so that the image is converted into a functional form $f(\boldsymbol{x})$, where $\boldsymbol{x}$ is a discrete picture element (pixel) in the grid and $f(x)$ is a discrete value, and is sometime called the image matrix, see Dougherty (1994). The digital image takes one of the following three formats: binary (1 bit), multiple levels of gray (for instance, 8 bits), or color, which indicates the amount of information from the physical analog image.

Since the sampling can be automated to some extent by electrical devices and computers in capturing, the main part of our digital image processing related to geometry modeling starts with a sampled (digitized and quantized) image stored in a computer. In the description below, we will confine ourselves to the gray scale image which contains 256 pseudo-gray shades (8-bits) which values ranging from 0 (white) to 255 (black).

\section{4 \\ Selecting}

Since the homogenization analysis requires the preparation of the numerical model of a unit cell that is a representative of the microstructure, an appropriate rectangular area of the image on the computer screen must be chosen. This area is regarded as a surface of the FE model of a unit cell and each pixel contained in it corresponds to a finite element in the surface. Therefore, the size of the area we can choose is dependent of the machine capability in which the FE analyses are made. We must carefully select the area section to be a desired model size by observing the number of pixels. The rectangular region selected this way is sometimes called the working image, see Berryman (1985). The region can be expanded or shrunk using a drawing software, e.g., Canvas ${ }^{\mathrm{TM}}$ by Deneba Software, if necessary, in order for it to contain the desired number of pixels. Although the true scale of this working image (e.g., microns/pixel) can be computed using functions in the software, e.g., the scale reference bar of the scanning electron microscopy (SEM), we do not use the scale since the microscopic problem does not require the physical scale. Figure 2 shows the selected digital image (400-by400 pixels, no scaling) of MMC using NIH image on a Macintosh personal computer. In the figure, there are some intermediate pixel values depending on the proportion of black and white in resolution cell and perhaps on the software we are using. The distribution of pixel values can be identified by a histogram of these values for the image. For instance, NIH image provides the function that constructs the histogram as shown in Fig. 3 for the present example. Using such information, we threshold the digital image data so that we would have a final configuration of the microstructure.

\section{5}

\section{Thresholding}

The thresholding is defined as "an image operation which produces a binary image from a gray scale image" in Haralick and Shapiro (1994). Furthermore, "the thresholding can produce a binary one on the output digital image whenever a pixel value on the input digital image is below a specified threshold level. A binary zero is pro- 


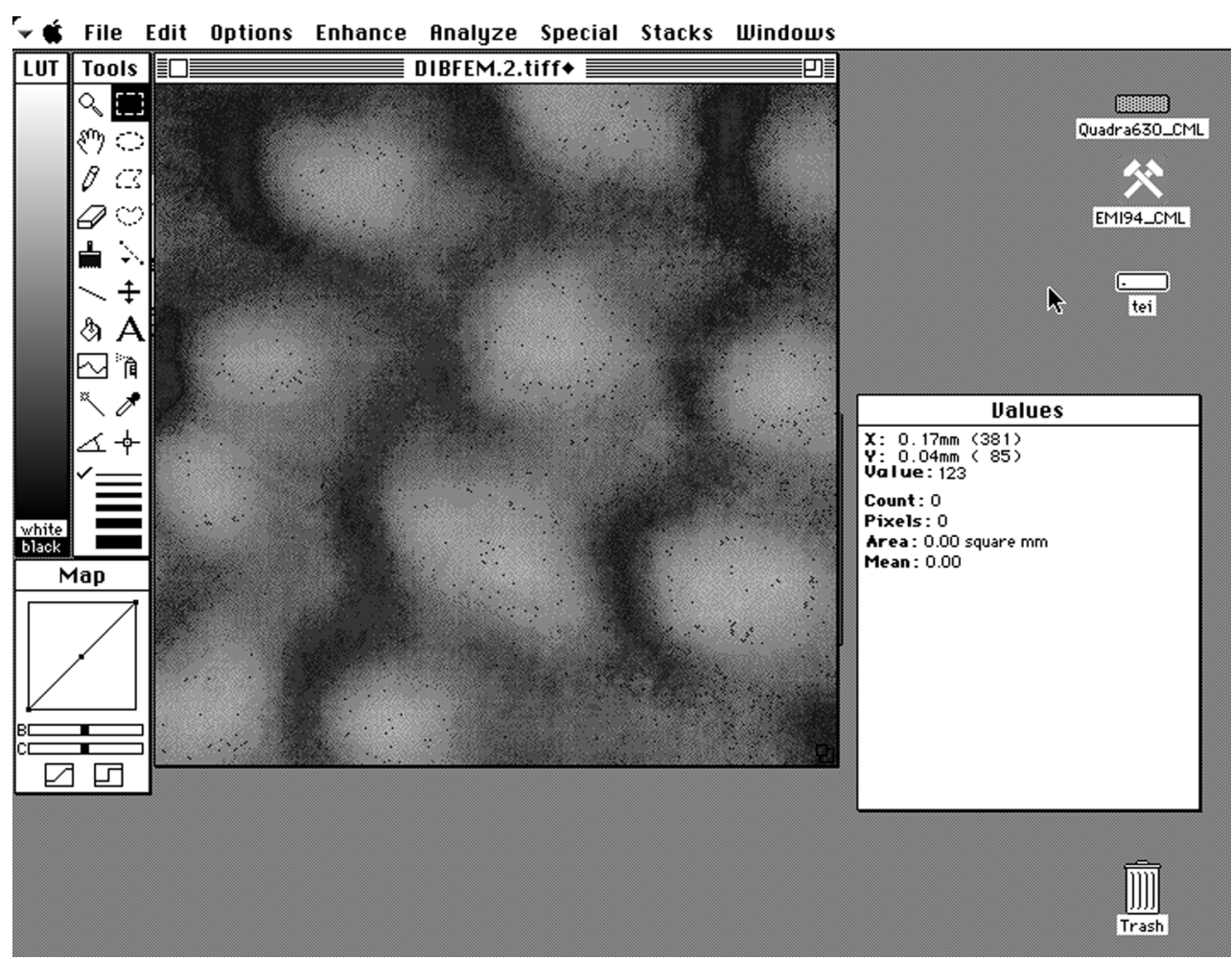

duced otherwise." Although a composite material is not always composed of two phases, we shall consider twophase composites for simplicity. Nonetheless, the definition can be easily extended by increasing the number of thresholding values.

The threshold values may be determined interactively by the operator. While the video display affords direct comparison of the thresholded image with the working image, the software enables us to modify or fix the thresholded images. Sine we have assumed that the phases of the composite could be distinctive in the original image, the thresholding is done by referring to the histogram on the video screen. If the original image has enough resolution and little noise, then the histogram can provide most of the information needed to choose the threshold value required for generating approximated geometry. Furthermore, if the volume fraction of each phase in the two dimensional image is given, the software may provide a function to calculate the ratio between pixel values, by which the threshold value is determined. On the other hand, if there is some noise, e.g., due to bad resolution of

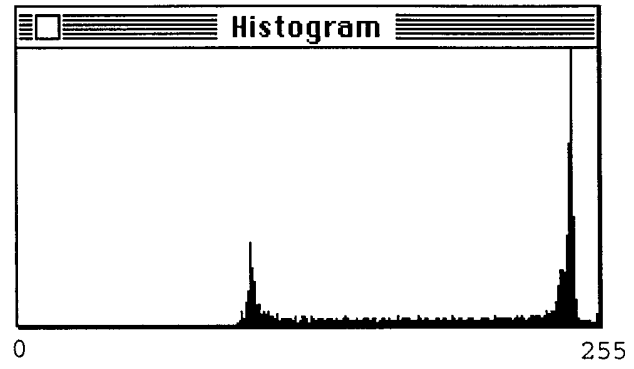

Fig. 3. Histogram of pixel values the captured image, it have to be eliminated manually by the drawing function in the software so that the desired geometry can be obtained. This additional operation is called adjusting.

Once a threshold value has been chosen, it is easy to convert the selected image into a binary image. If the obtained image does not seem to be a satisfactory representation of the original, new threshold values may be chosen until we are satisfied with the binary image. Figure 4 is the final form of the binary image mapping of the previous example.

\section{6}

\section{Exporting and stacking}

Now each pixel has either a binary zero or binary one in a digital computer and the binary data are exported into an ASCII file and transferred into a UNIX platform so that our computer program can read and recognize the data. This set of data is actually the prototype of our FE model, which is either 2D or 3D. If the analysis is two-dimensional, further operations are unnecessary and therefore it is passed to the FE analysis programs directly. If the analysis is three-dimensional, then each pixel is recognized as a volume element (voxel) and a necessary number of two dimensional ASCII files must be generated following the same procedure so that the totality can construct three dimensional unit cell. Here, the distance between successive two-dimensional images must be the same order as one pixel because the intermediate region is simply made by extruding one of these images. This step is called the stacking, the set of ASCII files that defines a 3D structure is called stack sequence, and can be incorporated into the computer program for the FEA. Figure 5 illustrates the stacking process schematically and Fig. 6 shows 


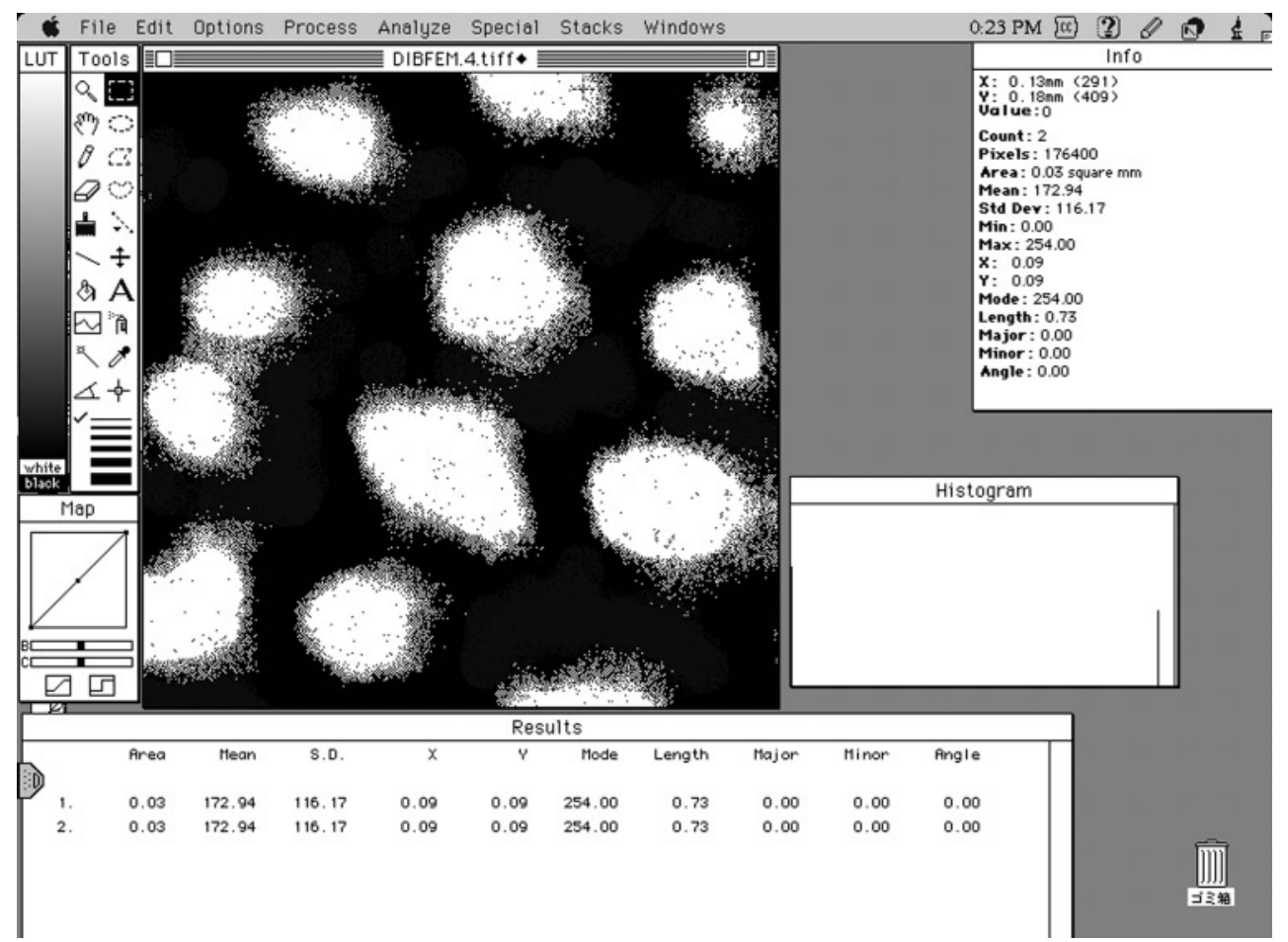

Fig. 4. Binary image after thresholding (and adjusting) the flow chart of DIB-FE modeling. Here we note that the order of the selecting and the thresholding can be changed depending on circumstances.

We will use the digital information in the FORTRAN programs written for geometry modification and the material pre-/post-processing in the homogenization method. To this end, the thresholding can alternatively be operated in the computer program which thresholds the digital image after the raw selected image is converted into ASCII

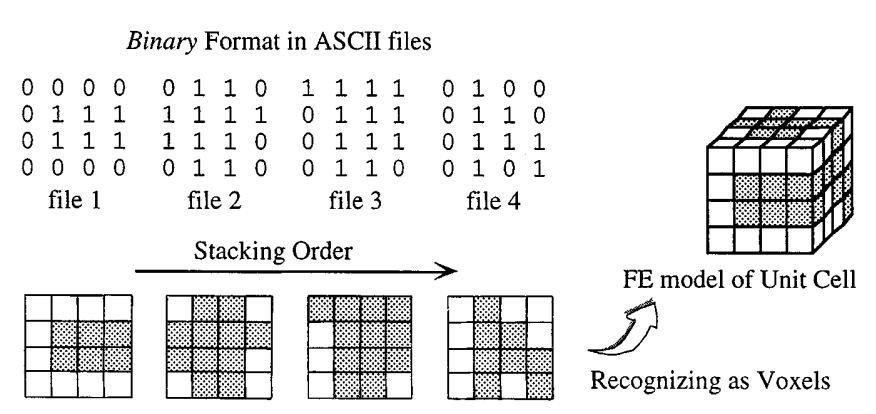

Series of Surfaces containing Pixels

Fig. 5. Stacking process for 3D structure

Capture and Sampling

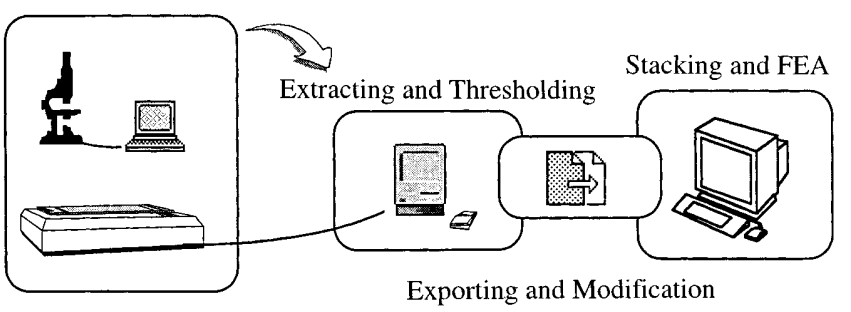

Fig. 6. Whole procedure of DIB modeling format; our computer program having those functions was named THOLDER. This program can also compute the volume fraction of each constituent for a given microstructural FE model by counting the voxels. A slight modification makes it possible to change the volume fraction of each constituent arbitrarily. The program having this function was named VFCHG. These operations will be discussed latter in the application of DIB-FE modeling.

\section{4}

\section{Digital image-based finite element method}

\section{1}

\section{Finite element procedure}

The ASCII files obtained by the series of image processes is the direct interpretation of the analog images which are two-dimensionally presented pictures of real composite materials. In fact, this is a great advantage because the procedure does not include any meshing manipulation such as defining coordinates and element connectivities, applying periodic boundary conditions and others, all of which are usually tedious tasks in FE geometric modeling. Here, let us explain how the digital images are utilized in FEA confining ourselves to three-dimensional cases.

Throughout the descriptions, we assume that we have had a proper set of material constants necessary for the analyses since they must have been obtained prior to the FE modeling.

The first step in DIB FEA is to recognize each voxel, which is actually a pixel in an image, as a finite element, accompanied by the stacking process of ASCII files. In an input file for a certain FORTRAN program, we specify the dimension, the model size, material properties, their ID's, and the number of ASCII files which define all the sections 
of 3D unit cell structure. Once the numbers of voxels in three coordinate axis directions are counted in the program, the size of one element in each direction is determined. This means that the geometry information of a hexahedoral FE mesh, e.g., the numbers of nodes and elements, element connectivities, can be automatically defined since all the element have the same size. Furthermore, even the periodic boundary condition can be automatically determined because the position of each boundary nodes is fixed. Also, each voxel value, which has been obtained by thresholding, is identified with the material ID's by specifying their correspondence in an input file. This indicates that each voxel value determines the corresponding type of material.

Although these are indeed all the processes of FE mesh generation, it is worthwhile to note that we do not need to store the element connectivity and coordinate values while running the program. That is, the information can be evaluated only if necessary since the size of an element is fixed. This feature not only saves great amounts of memory but is also advantageous in the FE algorithm that will be explained below.

Since all the elements have the same size, the number of element stiffness matrices that we actually need is reduced to the number of materials. Together with the automated evaluation of coordinates and connectivity, we can utilize the so-called element-by element (EBE) FEM with preconditioned conjugate gradient (PCG) method of node-bynode-type, see, e.g., Carey and Jiang (1986) for the basic idea. That is, we do not need the global stiffness matrix and, moreover, the element stiffness matrices, the number of which is equal to that of materials, are evaluated prior to the computation in PCG-routine. Each element stiffness matrix contributes to the force and direction vectors of PCG method during the iterations for convergence. Although these vectors with solution vectors still give a restriction on the FE model size, this method can handle a much larger number of elements than usual methods when we try to analyze one particular microstructure. The FE model used in the previous section has been read in HyperMesh of Altair Software, in which one can hardly observe each element, see Fig. 7.

In the DIB-FE modeling, we use much more elements than usual. This leads to some difficulties in storing and

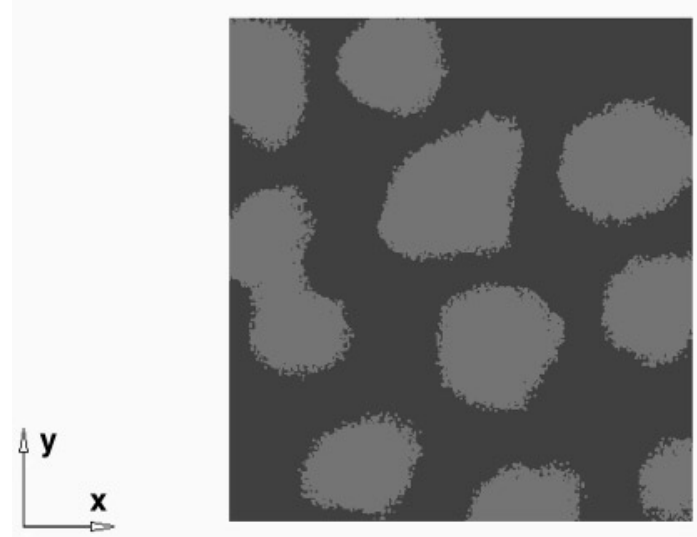

Fig. 7. FE model generated from digital image editing the result files of DIB-FEA, though this might not be the case if a supercomputer is utilized. Here, we consider ASCII data handling when using a UNIX EWS with ordinary memory and disk size. Once the material preprocessing part of the homogenization method is done by the DIB-FEM program, the results are written in an ASCII file, which contains the nodal values of characteristic deformation. As can be seen from the formulation of the microscopic problem, there are at least seven load cases for a 3D thermo-elasticity problem; six for mechanical strains and one for strain due to thermal expansion. As the number of elements is increased, the file size sometimes becomes so huge that storing the data may be impossible because of a lack of disk space and editing may be unmanageable in a screen text editor because of a lack of system memory. Therefore, we must store the data very carefully so that further editing is unnecessary and keep enough disk space available prior to the homogenization analysis by DIB-FEM to avoid the failure in storing. In order to reduce the file size, it may be helpful to store the result for each load case into one file. The features are also true for material post-processing (localization process) because we may have to handle the result files of the microscopic deformation/stress at several points in an overall structure, the size of which may also be huge.

\section{2 \\ Effects of resolution of captured images}

When the digitized FE mesh is used in the homogenization analysis, the results may not be realistic because an actual material boundary does not have such a zigzag shape as in Fig. 7, in which each particle is an assembly of cubicshaped elements. This irrelevant FE meshing is directly related to the resolution of the captured images since the FE model made in the DIB modeling is dependent on them. The purpose of this subsection is to investigate the effects of the resolution of captured images in conjunction with the FE approximation. It is expected that the unit cell model in the DIB modeling will provide more accurate estimation on both macro and microscopic variables if the resolution of captured image is improved.

In the investigation, we shall use an epoxy composite reinforced by glass fiber $\left(V_{f}=26.8 \%\right)$, each of whose material constants is given as follows: for epoxy, Young's modulus; $E_{m}=4 \mathrm{GPa}$, Poisson's ratio; $v_{m}=3.8$, and for glass, $E_{f}=76 \mathrm{GPa}, v_{f}=0.2$. As for the unit cell geometry, the basic structural element containing a single fiber is used because it is enough to justify the convergence of the microscopic variables with increasing the resolution, i.e., the order of FE approximation, in the IDB modeling. Thus, the analysis here will not lose the consistency with our purpose in this paper.

The stress values were calculated by applying (a) simple tension and (b) shear macroscopic strains $(0.1 \%)$, respectively, with $\Delta T=0 \mathrm{C}^{\circ}$. As Hollister et al. (1993) reported, there exist artifacts in numerical results obtained by the DIB-FEM, i.e., the stress concentration at the boundary between dissimilar materials, The stress contours in Fig. 9 also give such feature. In fact, the maximum stress, when using $25 \times 25$-pixel model, was $40 \%$ higher than that obtained by using the usual meshing although 

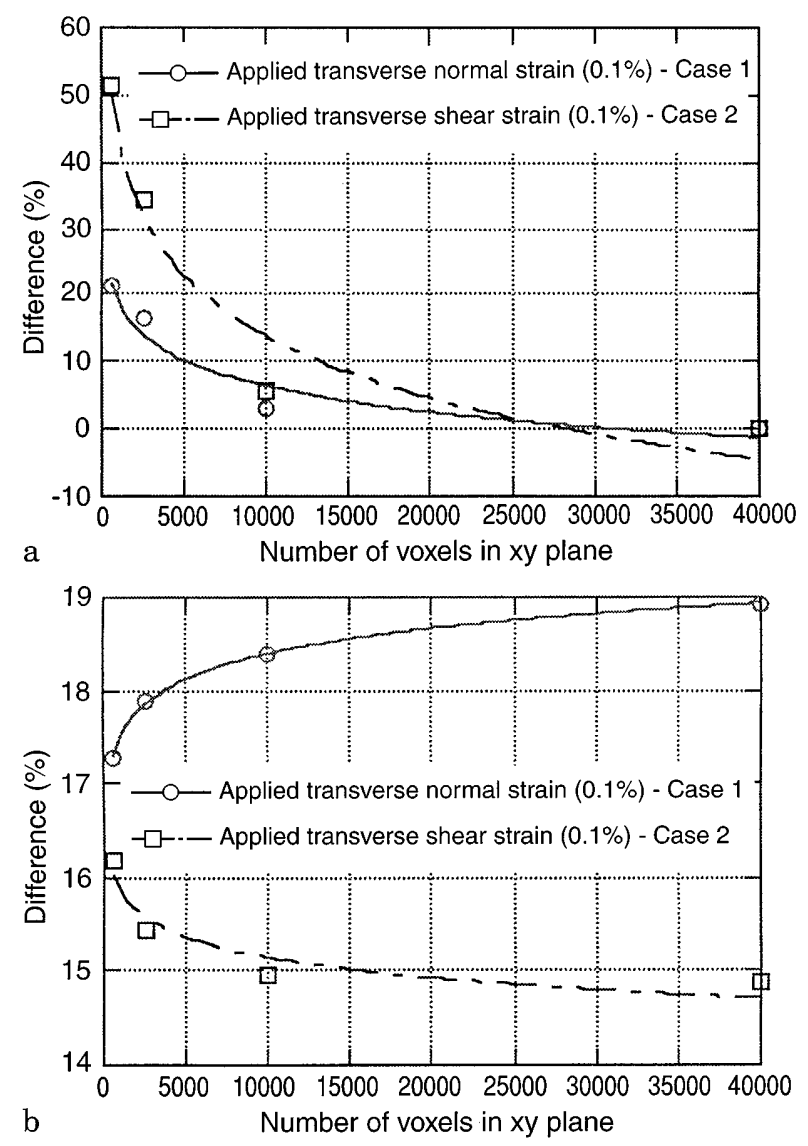

Fig. 8. Variation of microscopic stress with increasing number of voxels

the homogenized elasticity constants were almost the same.

Since it is difficult to discuss about the effect of resolution from Fig. 9, let us see the convergence trend of the microscopic variables. Figure 8 (a) and (b) show the variations of the microscopic maximum stress and the standard deviation in the contour images (Fig. 9), respectively, as the resolution is improved. From these figures, the microscopic variables become close to certain values as the number of elements increases. This fact justifies the following argument: if the resolution of the captured image is good enough to represent the accurate geometry of the inclusions, then the microscopic variables and also the macroscopic variables are accurately evaluated by the digitized mesh using the DIB modeling. In this particular material configuration, one-hundred pixels or above in $x y$-plane are needed for accuracy.

In conclusion, the resolution of the captured image must be as good as possible. However, the better the resolution, the larger the number of finite elements, and therefore much larger memory size of a computer is required to store the solution vector, which is used for nodal values of characteristic functions. Nonetheless, this modeling technique is extensively utilized for all the simulations by the homogenization method in this paper.

\section{5}

\section{Justification for RVE analysis}

\section{1}

\section{RVE analysis}

The introduction of a representative volume element (RVE) is the first step in the RVE analysis (Bear (1967)), which implies that we adopt the continuum approach as a mathematical modeling method, prior to the numerical modeling. The RVE analysis assumes the capability to pass from the microscopic level, at which each phase is regarded as a continuum, to macroscopic one, at which volume averaged quantities, i.e., effective material properties capture macroscopic phenomena in the vicinity at any point. Therefore, the construction of a continuum model of a composite material imposes certain restrictions on the size of the RVE. Foremost is the requirement that the values of all effective material properties at any point in the overall structural domain be single values functions of that position and of time only, independent of the size of the RVE. Since the asymptotic homogenization method also takes the concept of RVE analysis in general sense, we shall justify the feasibility of the present DIB modeling and also clarify the advantage of it in this context.

In the DIB modeling, this study is related to the capture and selection process described above. Denoting the characteristic dimension of the captured domain by $l$, (say

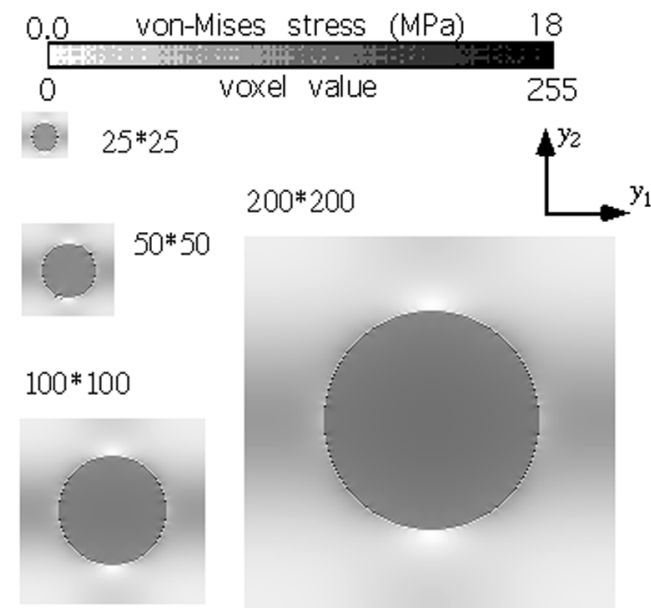

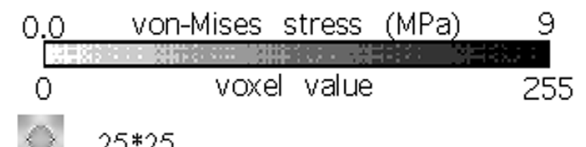

$25 * 25$

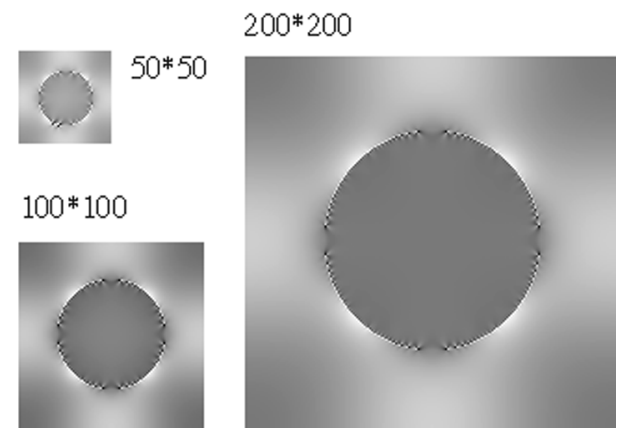

Fig. 9. Microscopic von-Mises stress distribution. a Case 1, b Case 2 
edge length of a micrograph), and the length characterizing the microscopic structure of each constituents by $\delta$ (say, the fiber diameter for fiber-reinforced composites), there is the following relationship:

$l \geq \delta$

where $\delta$ actually sets a lower limit to the size of RVE, under which a basic structural configuration cannot be a representative one. Also, denoting a characteristic length for a macroscopic domain for a composite material by $L$, we postulate the following relation so that the RVE can be meaningful:

$L \gg l$

where $L$ is the upper limit, over which averaged quantities does not make sense. Thus the characteristic length $\varepsilon$ of the selected domain, namely RVE (unit cell) domain, must be comparable with $l$ and is bounded by these upper and lower bounds as follows:

$L \gg l \sim \varepsilon \geq \delta$

\section{2}

\section{Justification of DIB modeling}

Here we shall present the results from the material pre-Ipost-processing of the homogenization analysis in order to show how DIB modeling fits to the micromechanical RVE analysis. Given a micrograph of a MMC together with material properties of each constituent (which are the same as in the previous 3), we select the unit cell from a sampled image as presented in Fig. 10 and assume the material constants as shown in Table 1 . We may expect that we will obtain the same effective (homogenized) material properties when the arbitrary size of RVE (unit cell) is selected from appropriate regions, A, B,

Table 1. Material properties for MMC (NiAl-Cr)

\begin{tabular}{llll}
\hline & $\begin{array}{l}\text { Young's } \\
\text { modulus (GPa) }\end{array}$ & $\begin{array}{l}\text { Poisson's } \\
\text { ratio }\end{array}$ & $\begin{array}{l}\mathrm{CTE} \\
\left(\times 10 \mathrm{e}-5 /{ }^{\circ} \mathrm{C}\right)\end{array}$ \\
\hline $\begin{array}{l}\text { NiAl-Matrix } \\
\text { Cr-Fiber }\end{array}$ & 177 & 0.30 & 1.60 \\
\hline
\end{tabular}

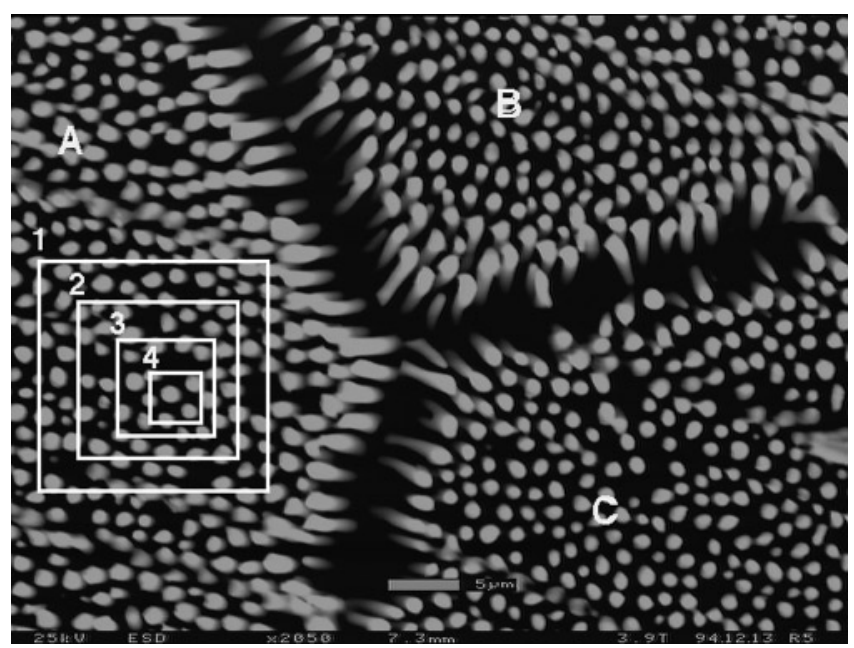

Fig. 10. Sampled image with several areas to be selected

or $\mathrm{C}$ in the figure. Since we have only one captured image, let us assume here that the fibers are straight and aligned perpendicular to the image so that the three dimensional geometry could be constructed by extrusion. Although numerical models of defects shown in the figure can also be made by the DIB modeling technique, we will not consider these issues since they seem to be beyond the present discussion.

Choosing the region A in Fig. 10, let us assume that the thresholding has been successfully done for the whole region. Then different selections of unit cells give different FE models and therefore different sets of homogenized properties by the material pre-processing, i.e., the homogenizing. It should be noted that one fiber of each model has the same numbers of voxels, although each selected image contains a different number of voxels. This guarantees the equivalency in the orders of FE approximation by digital images and makes the comparison meaningful. Table 2 compares each of the homogenized material properties to the others in the form of engineering constants and shows that the differences appear to be small. As can be seen from the table, all the unit cell models are equivalent in the sense that they provide almost the same values of effective (homogenized) material properties, independent of the size of the selected area.

Table 2. Homogenized material properties of MMC (NiAl-Cr) for different selected areas

\begin{tabular}{lcccccc}
\hline$* *$ & $25^{*} 25$ & $50^{*} 50$ & $100^{*} 100$ & $200^{*} 200$ & idealized $^{\text {analytical }^{* *}}$ \\
\hline E1 & 197.58 & 197.54 & 197.48 & 197.42 & 197.43 & 195.91 \\
E2 & 197.39 & 197.37 & 197.26 & 197.26 & 197.43 & 195.91 \\
E3 & 200.46 & 200.40 & 200.39 & 200.38 & 200.41 & 200.40 \\
G23 & 75.97 & 75.99 & 75.95 & 75.95 & 75.90 & 75.35 \\
G31 & 76.07 & 76.02 & 76.06 & 76.06 & 75.90 & 75.35 \\
G12 & 75.65 & 75.65 & 75.66 & 75.67 & 75.42 & 75.35 \\
a11 & $1.3946 \mathrm{E}-5$ & $1.3952 \mathrm{E}-5$ & $1.3957 \mathrm{E}-5$ & $1.3968 \mathrm{E}-5$ & $1.4007 \mathrm{E}-5$ & $1.4794 \mathrm{E}-5$ \\
a22 & $1.3980 \mathrm{E}-5$ & $1.3983 \mathrm{E}-5$ & $1.3988 \mathrm{E}-5$ & $1.3993 \mathrm{E}-5$ & $1.4007 \mathrm{E}-5$ & $1.4794 \mathrm{E}-5$ \\
a33 & $1.3475 \mathrm{E}-5$ & $1.3481 \mathrm{E}-5$ & $1.3482 \mathrm{E}-5$ & $1.3843 \mathrm{E}-5$ & $1.3480 \mathrm{E}-5$ & $1.1920 \mathrm{E}-5$ \\
\hline
\end{tabular}

${ }_{* *}^{*} \mathrm{GPa}$; for elasticity constants, $/{ }^{\circ} \mathrm{C}$; for CTE

** The number of voxels in $x y$-plane

${ }^{* * *}$ Analytical Results implies: the results obtained by Halpin-Tsai equation (emperical parameter $=0.3$ ) for engineering elasticity constants and the relation of Schapery for CTE (Halpin 1992) 
Thus we can conclude that the DIB modeling is feasible enough to evaluate the effective material properties.

It is worthwhile to see the differences in homogenized properties between the FE models made by DIB modeling and an idealizing FE model, see Fig. 11. Table 2 also compares the homogenized material properties of those two kinds of models together with the analytical estimates (Halpin 1992). As can be seen from the table, they are slightly different. This difference may be caused by the random nature of the models made by the DIB modeling and is related to the mophology in the unit cells. In addition, although the orthotropy was enforced in Table 2, the homogenized elasticity constants did reveal an anisotropic nature due to coupling between the transverse stiffnesses while the structural configuration of the idealized one gives an orthotropic one. Although this result may not be applied to whole domain of the composite, we can say that it is true atleast for the local region where this image is captured. In contrast, the coupling between longitudinal stiffnesses is not observed, since we have assumed that the fibers were aligned perpendicular to the given two-dimensional image. This artificial de-coupling is induced by the captured images. The dependence on captured image is disscussed in the next subsection from another viewpoint.

Furthermore, as can be seen from Fig. 12 we can find considerable differences in the microscopic stress fields obtained in the material post-process, i.e., the localization, when comparing the results from the idealized model and those obtained from the DIB modeling $(100 \times 100$ voxels $)$. Here these two results were obtained by applying the same average strain field whose components are given by

$$
\boldsymbol{e}\left(\boldsymbol{u}^{0}\right)=\left[\begin{array}{ccc}
1400 & 2.5 & 0.0 \\
2.5 & 1400 & 0.0 \\
0.0 & 0.0 & 1400
\end{array}\right] \times 10^{-6}
$$

and the temperature change $\Delta T=-100^{\circ} \mathrm{C}$ in equation (15). It is obvious that the stress distribution in the model generated by the DIB modeling technique is more realistic though the orders of FE approximation are different because the model approximates the actual fiber alignment. Therefore, a qualitative and quantitative study of the micromechanical behavior of composite materials is pos-

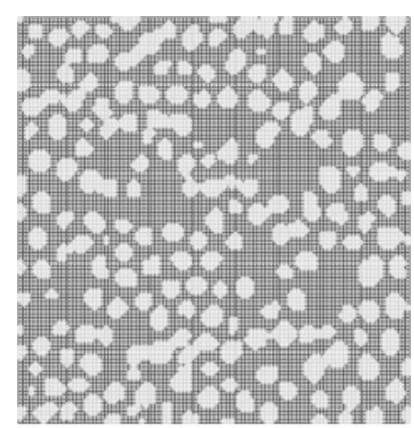

a

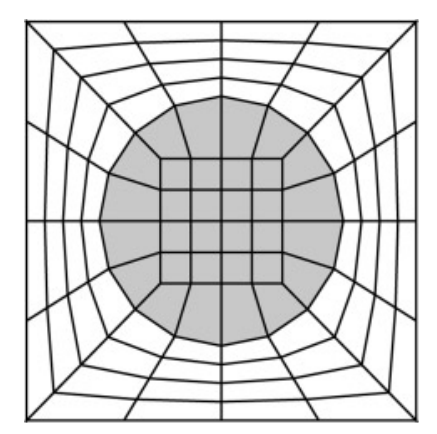

b
Fig. 11. DIB model and idealized FE model of MMC. a Digitized $(100 \times 100 \times 4$ voxels $)$ model, b Idealized (384 HEXA8 elements) model
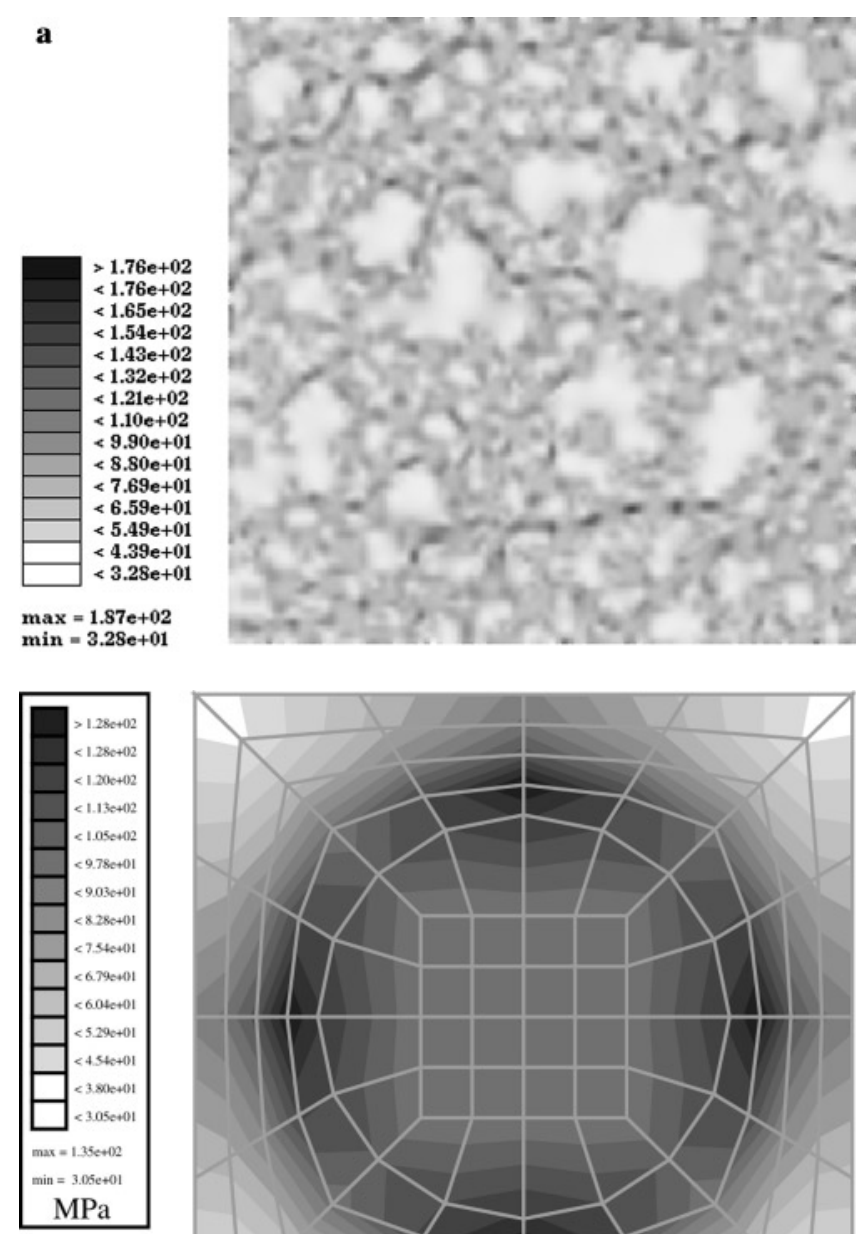

b

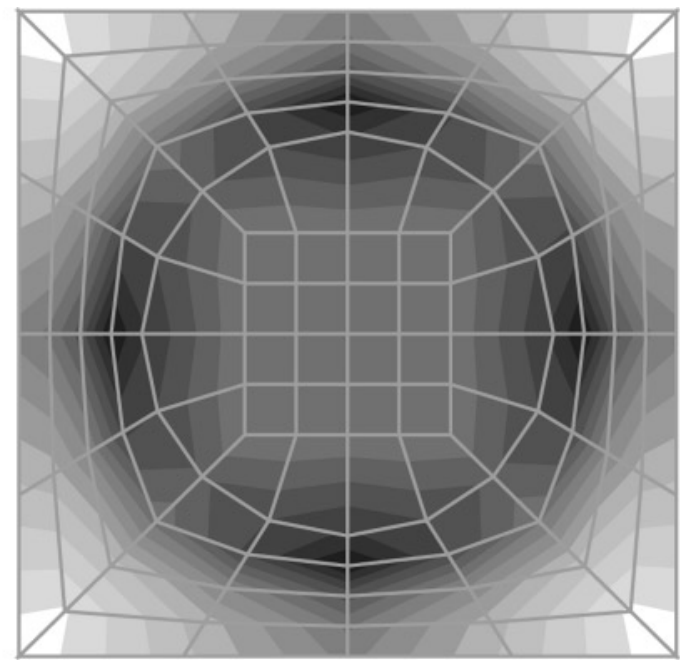

Fig. 12. Localization results of DIB model and idealized model. a von-Mises stress $(\mathrm{MPa}), \mathbf{b}$ von-Mises stress $(\mathrm{MPa})$

sible. Although the presence of some disadvantages has been pointed out, some of which are reported by Hollister et al. (1994), e.g., fluctuating stress values near the boundary of dissimilar materials, the numerical example presented in subsection 4.2 suggests one of the solutions.

As a final note of this section, the localization results can be converted into images, namely collections of discrete pixel values and analyzed by utilizing the functions in image processing software. In fact, the stress result in Fig. 12 (a) was an image in Adobe Photoshop and modified so that the element boundaries are blurred. An example of an image analysis in the software is given in Fig. 13. Using such functions in image processing software, we can carry out the pixel based evaluation of the stress analyses.

\section{3}

\section{Comment on dependence on captured images}

It is apparent that if we use different images captured from the same camera-angle, the models by DIB-FE modeling are different, but the homogenized material properties obtained from them should be the same if the fabrication 


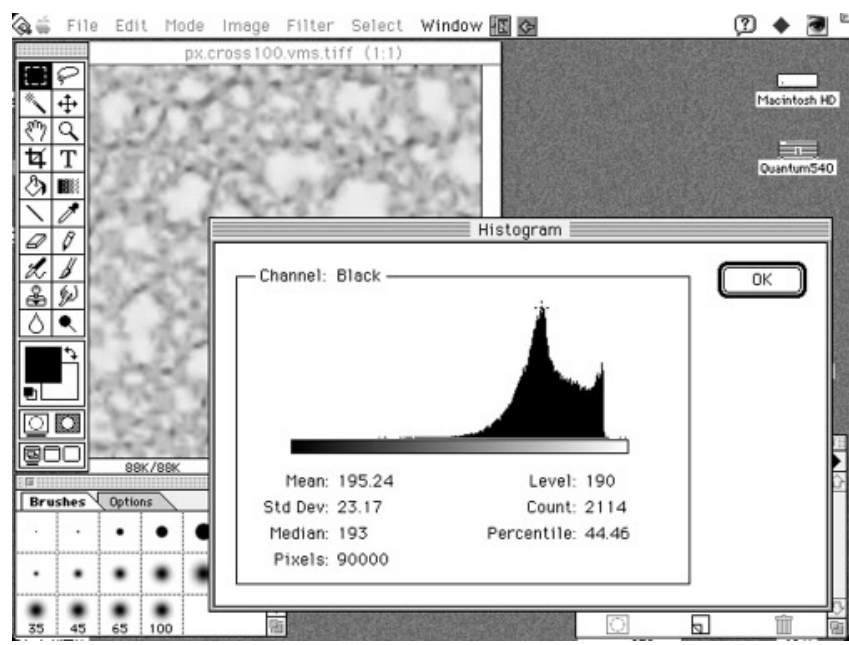

Fig. 13. Image processing and analysis in post-process of DIB-FEM

is performed in the same way. However, when the micrograph of the same MMC shown in Fig. 14 is given, what would become of the homogenization results by DIB-FEM? We shall comment on this issue.

In order to examine the influence of the orientation in which the capture is processed, we shall first explain how to make the FE model from this kind of image. The only difference from the previous example lies in the thresholding process in the DIB modeling. Although it seems difficult to make a three-dimensional structure from the given plane image, it is relatively easy if we assume the fibers have circular cross sections because the original image has 256-scales which enables the perspective thresholding technique. That is, a unit cell FE model which contains several circular fibers can be tailored by generating several two-dimensional digital images, each of which has an individual threshold value. The FE model made in this way is shown in Fig. 15 and the homogenized properties are given in Table 3. The major components of the elasticity constants almost coincide with those obtained from the models in Fig. 10. It might be possible to

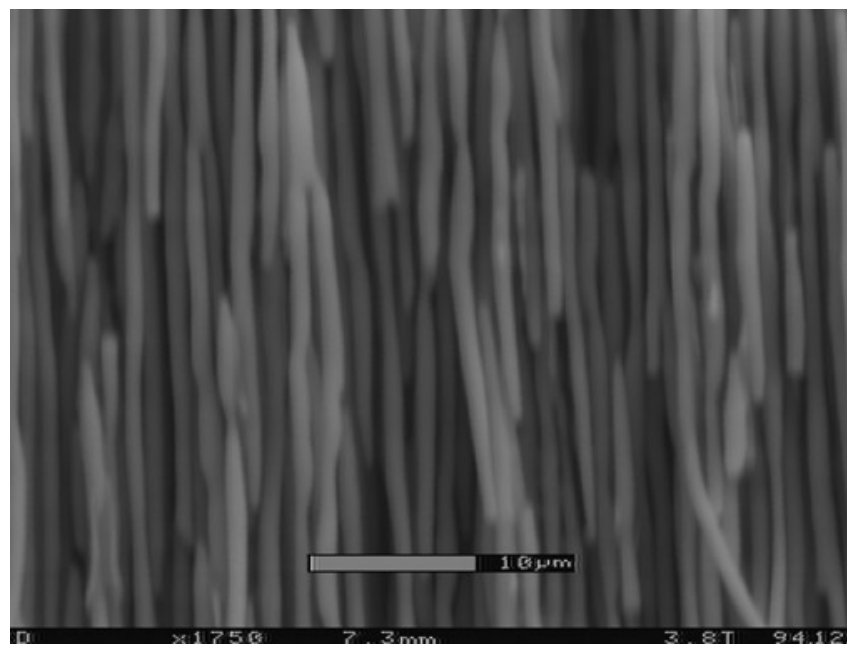

Fig. 14. Micrograph of different view

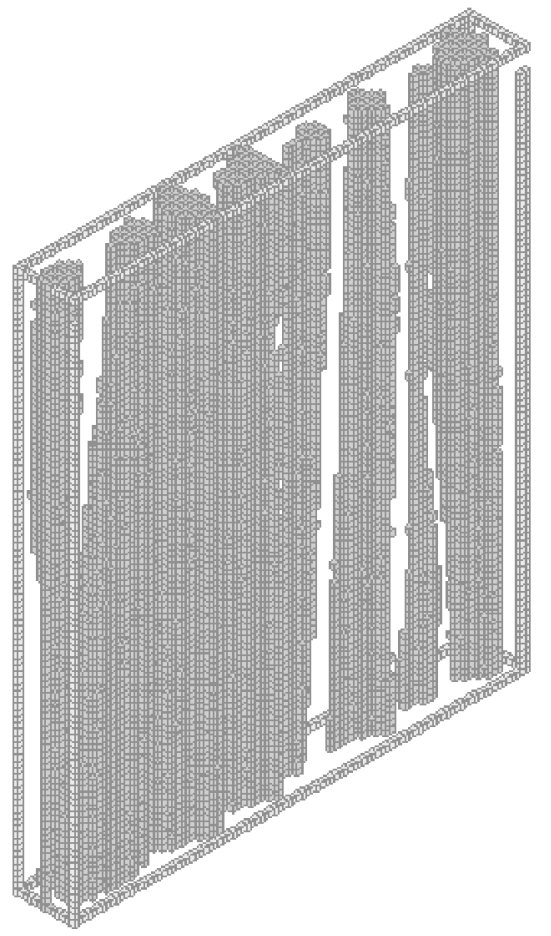

Fig. 15. Digitized FE model obtained from micrograph of different view

combine those two results to obtain a more accurate estimation of the homogenized stiffness. Note here that the coincidence of the results was brought about by proper images and that this is not always the case. In fact, the DIB modeling strongly depends on the captured image and must require more images which can lead to a $3 \mathrm{D}$ structure, in most of the cases.

\section{6}

\section{Applications of DIB modeling to micromechanical studies}

\section{1}

\section{Introductory remarks}

The objective of this section is to illustrate how digital image processing is applied to the $3 \mathrm{D}$ micromechanical studies of composites by the homogenization method. Since the digital images yield ASCII files, each of which represents a two-dimensional section of the FEA model, changing the microstructural geometry configuration implies changing the voxel values. This simple formula provides a significant convenience in modifying the $\mathrm{FE}$ model of a unit cell and therefore in the design process. We shall introduce here two examples of digital image manipulations; one is for the technique to change vlolume fractions and other is for the technique to virtually construct a three-dimensional FE model of a unit cell. In both

Table 3. Homogenized engineering constants. GPa; for elastictiy constants, $/{ }^{\circ} \mathrm{C}$; for $\mathrm{CTE}$

\begin{tabular}{lllllllll} 
E1 & E2 & E3 & G23 & G31 & G12 & a11 & a22 & a33 \\
\hline 198 & 200 & 198 & 76.1 & 75.5 & 76.1 & $1.39 \mathrm{E}-5$ & $1.35 \mathrm{E}-5$ & $1.39 \mathrm{E}-5$ \\
\hline
\end{tabular}




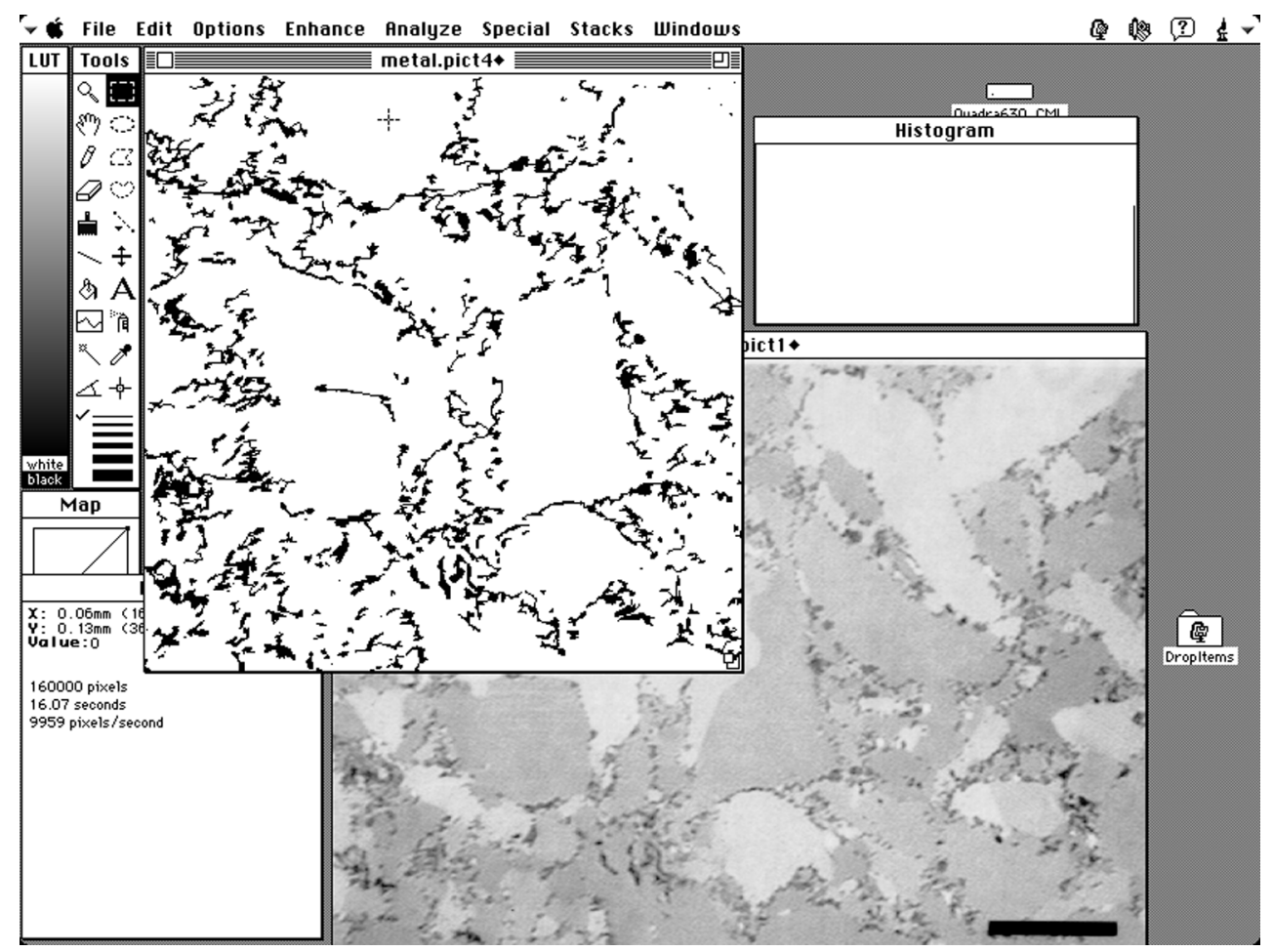

Fig. 16. Sampled and selected images of MMC $\left(\mathrm{MoSi}_{2}-\mathrm{SiC}\right)$ examples, a metal matrix composite (MMC) which is composed of $\mathrm{MoSi}_{2}$-matrix and $\mathrm{SiC}$-inclusion. Figure 16 shows the thresholded and adjusted binary image along with the sampled image, which has $400 \times 400$ pixels. The material properties of these constituents are given in Table 4.

\section{2}

\section{Simulation of changing volume fractions}

When we are considering the mechanical behavior of composite materials and eventually the microstructural design, the geometric configuration of the FE model of a unite cell is subject to change. In this context, we may have to prepare different specimens of different volume fractions in order to find the mechanical characteristics of the composite as a function of the volume fraction of the inclusion. However, not only it cost much but also the consistency in microstructural geometry configurations between the specimens is questionable because, as can be seen Fig. 16, the reinforcement (inclusion) is scattered in a random manner which comes from its fabrication methods such as solid or liquid state processing and deposition. Therefore, these random nature cannot be neglected by idealizing the geometric configurations in the microstructure. The original FE model of the unit cell has to be appropriately modified, keeping the original geometry

Table 4. Material Properties for $\mathrm{MoSi}_{2}-\mathrm{SiC}$ composite

\begin{tabular}{llll}
\hline & $\begin{array}{l}\text { Young's } \\
\text { modulus }(\mathrm{GPa})\end{array}$ & $\begin{array}{l}\text { Poisson's } \\
\text { ratio }\end{array}$ & $\begin{array}{l}\mathrm{CTE} \\
\left(\times 10 \mathrm{e}-6 /{ }^{\circ} \mathrm{C}\right)\end{array}$ \\
\hline $\mathrm{MoSiO}_{2}$-matrix & 400 & 0.25 & 5.0 \\
$\mathrm{SiC}-$-inclusion & 450 & 0.20 & 8.1 \\
\hline
\end{tabular}

configurations as much as possible. We present below the technique to change the volume fractions of the inclusion in conjunction with the microstructural design according to the thermal mismatch in microscopic stresses.

The outline of the technique is as follows: we first assume that the image $(100 \times 100$ pixels $)$ has been selected and exported into an binary ASCII file (binary one for SiC and zero for $\mathrm{MoSi}_{2}$ ) of the unit cell. Here the same image is used to construct the three-dimensional structure by extrusion. It is also assumed that the additional particles of $\mathrm{SiC}$ always come to the interface with $\mathrm{MoSi}_{2}$ if more inclusions are scattered so that volume fraction of $\mathrm{SiC}$ is increased. Then the modification of the unit cell geometry is straightforward using the computer program, namely VFCHG, and the ASCII file. First we read the ASCII file as an input, recognize each voxels as a finite element and tell the program our desired value of volume fraction of SiC. Secondly a pseudo-random number from a seed value is generated in VFCHG and then the value is identified with the spatial position of a voxel in the unit cell model. Thirdly, according to the voxel value and neighboring voxel values, we check if it is on the boundary of the two dissimilar materials or not; then if it is so, voxels surrounding that pixel is set to binary one (which corresponds to inclusion, $\mathrm{SiC}$, in this case). If the voxel is not on the boundary, a new pseudo random is generated following the same procedure up to this stage. In the last step, the volume fraction of the inclusion is re-calculated and the value is compared to the input-value of volume fraction. The whole procedure is repeated until the re-calculated value is equal or the error set by the user is close enough to the desired value. The procedure is presented schematically in Fig. 17 It should be noted that the seed value depends on the operating system (OS) on which the program (VFCHG is running and therefore so does the 


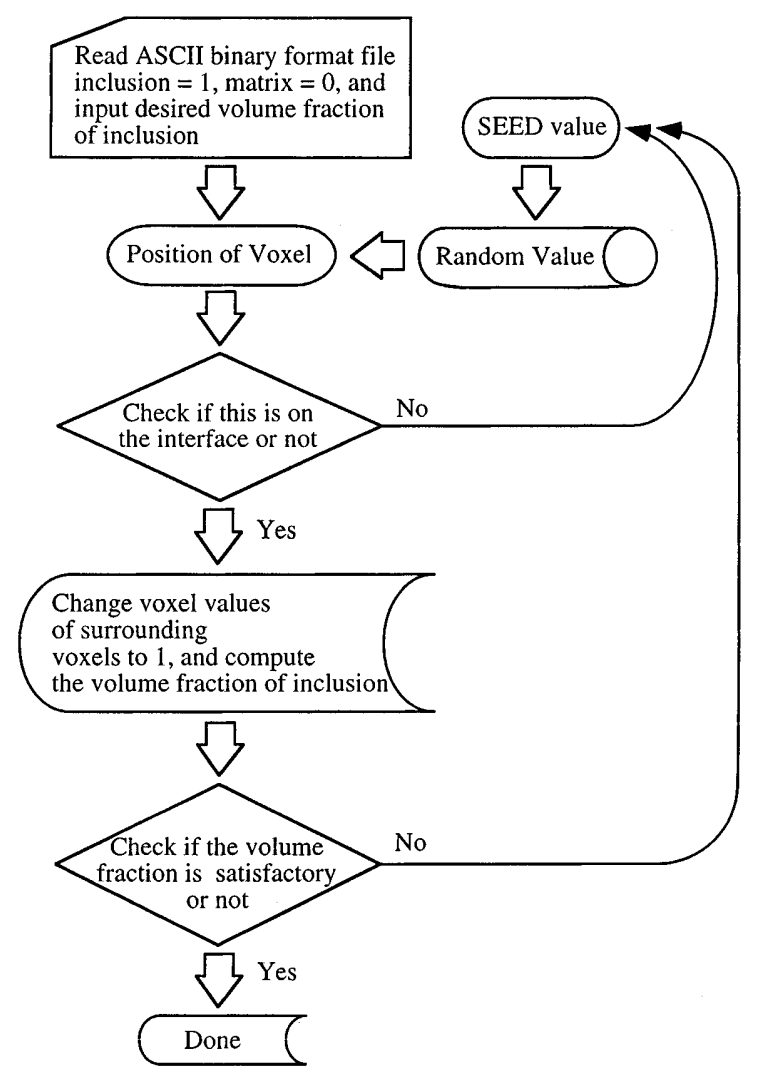

Fig. 17. Flow chart for changing volume fractional proportion

random value. Although the resulting microstructure may differ from the one that was generated on a different OS or different occasion on the same OS, it is reasonable to expect that the geometrical configuration is almost the same so that the homogenization analysis makes sense.

The systematic modeling technique by digital imaging made these estimates possible without any difficulty. Figure 18 shows the unit cell models that were obtained by this procedure. The localization results are presented by applying the temperature change $\Delta T=-300 \mathrm{C}^{\circ}$ and no global strain. These models also provide three charts shown in Fig. 19 and 20 that relate homogenized elasticity

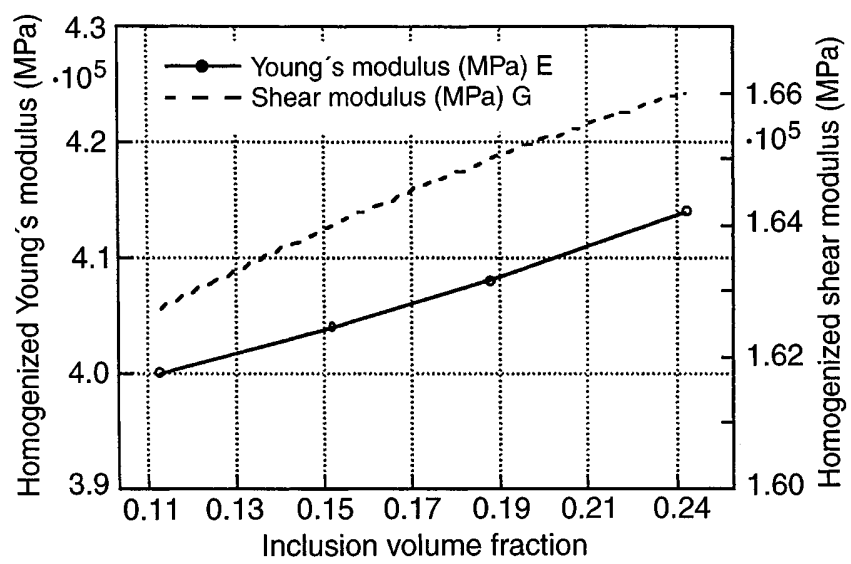

Fig. 19. Volume fraction (of $\mathrm{SiC}$ ) vs. homogenized elasticity constants

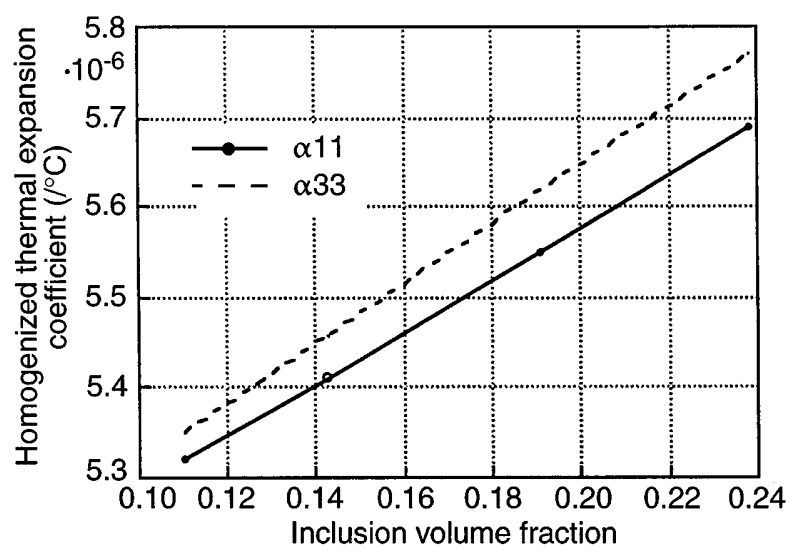

Fig. 20. Volume fraction (of $\mathrm{SiC}$ ) vs. homogenized CTE

constants and thermal expansion coefficients to the volume fraction of SiC, and Fig. 21 shows the thermal stresses averaged in each phase and the thermal mismatch between two phases versus the volume fraction of $\mathrm{SiC}$. Note that the former is related to macroscopic responses and the latter to microscopic ones. Therefore, we are interested in the optimal volume fraction referring to both the homogenized properties and the thermal mismatch, both of which
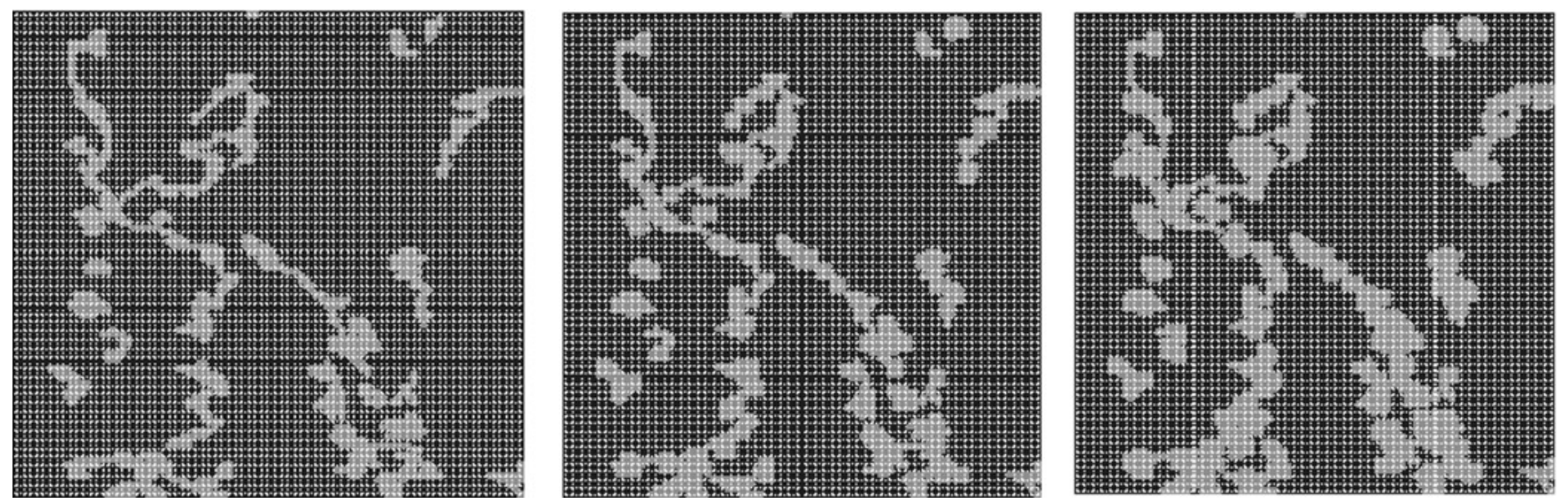

Fig. 18. FE models $(10,15,20 \%$ inclusion) generated by digital image with VFCHG 


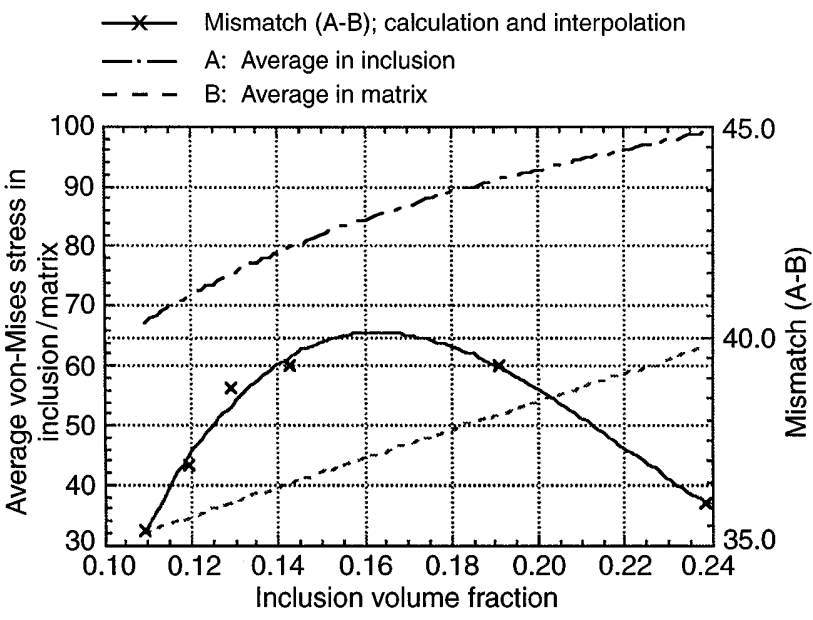

Fig. 21. Volume fraction (of $\mathrm{SiC}$ ) vs. mismatch in microscopic thermal stress (Stress values are averaged within the same material)

can be the objective functions of our design optimization problem. Since we are now concerned with the DIB modeling, we do not discuss this issue in detail and leave it for a later opportunity. It should also be noted that the simulation is reliable enough in the sense that we had a similar geometric configuration, i.e., a similar pattern of inclusion scattering.

\section{3}

\section{Virtual realization of three-dimensional microstructure}

All the three-dimensional (3D) FE models of unit cells that we have used so far were obtained from a plane image by extruding in the direction perpendicular to the image except in the example shown in Subsect. 5.3. As mentioned before, the sequential stacking of two-dimensional (2D) images can construct the actual 3D unit cell. That is, as a premise of generating a 3D structure by DIB modeling, we have to have enough data concerning the $3 \mathrm{D}$ geometric information. However, regarding the given composite that has a random nature, we have only one captured image. It is not only costly but almost impossible to prepare such a set of additional captured images which section the actual 3D structure because the required thickness of one section is too thin to capture. Although the sectioning devices such as CT-scanners are the most promising, another alternative way must be found in order to investigate the true 3D characteristics of the microstructure. We now propose a novel methodology below although the idea itself is quite simple.

Using the 2D geometric information, the idea of triangulation in the digital image processing is utilized. It is quite natural to assume that each projection of the $3 \mathrm{D}$ structure into the three coordinate planes is identical to the given $2 \mathrm{D}$ image. The we can determine all the 3D spatial positions by taking product of each voxel value in the projected images so that we can virtually attain the 3D image. Since we have assumed that the image data contain only binary ones or zeroes, the mutual multiplication also gives binary ones and zeroes. This multiplication serves as a function to change to attribute of a certain voxel value to the other. Denoting $f(\bullet, \bullet, 0)$ by the pixel value of each projected image, the functional form is defined as follows:

$F\left(x_{1}, x_{2}, x_{3}\right)=f\left(x_{2}, x_{3}, 0\right) \times f\left(x_{3}, x_{1}, 0\right) \times f\left(x_{1}, x_{2}, 0\right)$

where $\left(x_{1}, x_{2}, x_{3}\right)$ is the position of a voxel and $F$ is a voxel value of the virtual $3 \mathrm{D}$ image. Let us assume that we have stored the ASCII binary data of the 2D image into an array $\operatorname{mvl}(*, *, 1)$. Then, denoting the number of pixels in the coordinate axis directions by npix, npiy, and npiz, respectively, the image product defined by (20) can be stored into a new array mv2(*,*,*) by the following FORTRAN program:

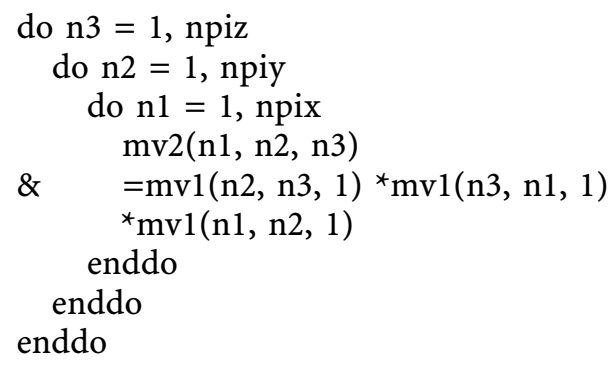

If we used 8-bit format file as an input, a new distribution of 24-bit map would be obtained. Then the thresholding technique enables the creation of several different 3D images. Moreover, if we replace the last two functions in formula (20) by the vector I whose components are all ones, the extruding of a $2 \mathrm{D}$ image is also represented by a functional form such that

$F\left(x_{1}, x_{2}, x_{3}\right)=f\left(x_{1}, x_{2}, 0\right) \times I\left(x_{3}\right)$

by which the extruded 3D image is derived.

If the procedure is done so that the volume fraction of the inclusion would be almost equal to the known value, the model can be recognized as a $3 \mathrm{D}$ realization of the actual microstructure of composite. However, there is no guarantee that the resulting 3D structure has the same volume fraction as the original. Therefore, we have to adjust it by using the VFCHG again. The unit cell derived in this way is presented in Fig. 22 along with the localization result whose stress computed by applying no strain and temperature change $-300 \mathrm{C}^{\circ}$. Although it is virtual, the results can simulate the mechanical behavior of the unit cell. If we have three mutually perpendicular 2D images, the result would be more realistic.

\section{4}

\section{Additional comments on the applications}

Recall that we are looking for the realistic geometry model by utilizing the digital image processing and eventually the micromechanical characteristics of composite materials along with the homogenization method. Although the applications presented here appear unrealistic, we assert that they are realistic, because the real 2D images, to which the image operations were applied, were used to derive the 3D geometry models. The models presented here can be the candidates of real unit cells, which elucidate the mechanical behavior of microstructure. We cannot prove this, but there is no way to deny it. 

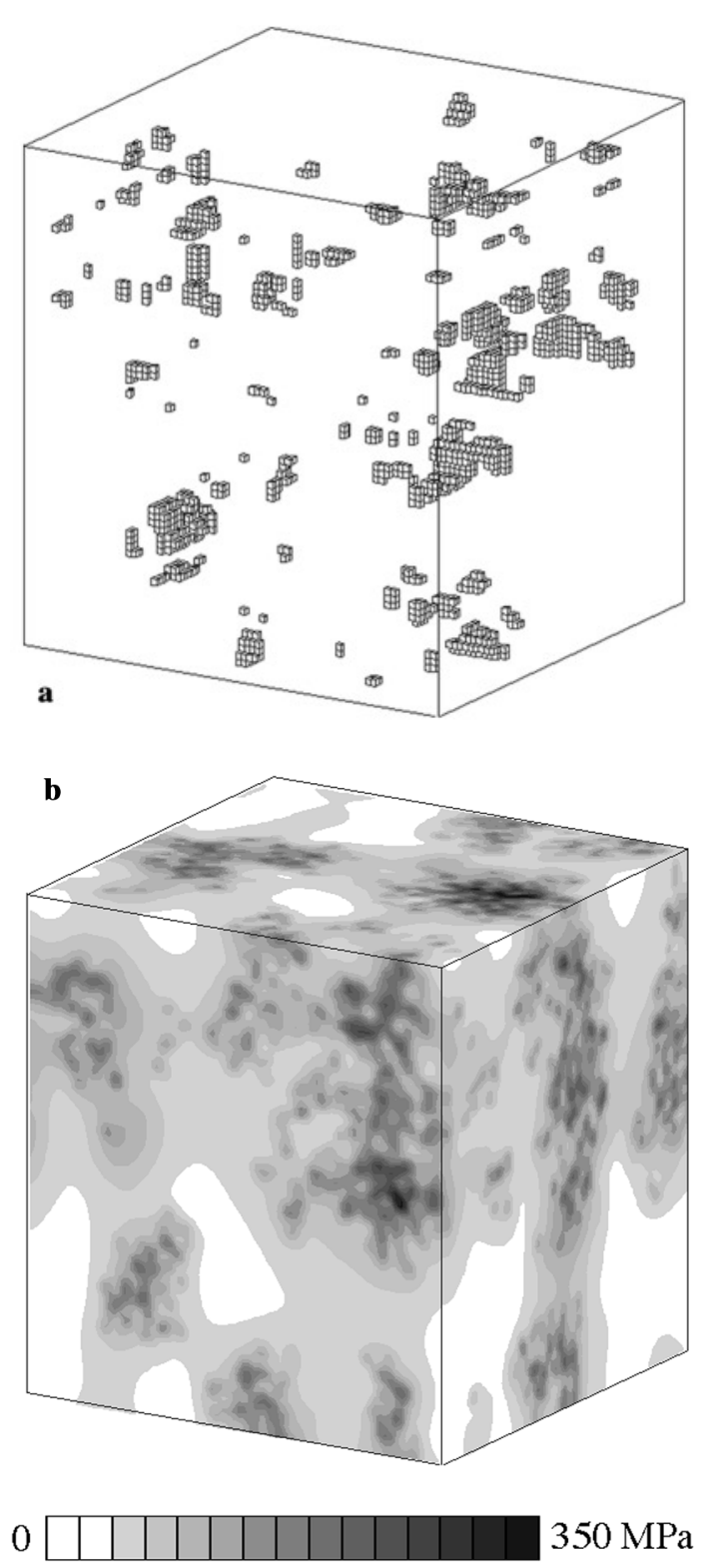

Fig. 22. Digitized FE Model by 3D realization with localization results. a Digitize FE Model of unit cell, $\mathbf{b}$ Microscopic von-Mises stress distribution

Although we have emphasized the usefulness of the digital images in numerical modeling it seems worthwhile to discuss the role of digital images from another viewpoint. Indeed, it is quite meaningful that we can determine $3 \mathrm{D}$ geometric configuration of the microstructure in the quantized form. In the formula (20) or (21), we recall that the quantization described in Subsect. 3.3 provides an image matrix $f(\bullet, \bullet, 0)$ which corresponds to the pixel value of the $2 \mathrm{D}$ image. The necessary operation is to fill the $x_{3}$-component to form the $3 \mathrm{D}$ structure and/or to change the pixel values. If we think of the inverse of digital images processing, it is possible to obtain the real composite media from the image. That is, given desired material characteristics, we determine the geometric configuration of the microstructure by the homogenization method, see, for example Terada et al. (1996). The unit cell derived by this inverse problem can be visualized and stored in our computers by some morphological operations. By the appropriate manufacturing technique, the digital images can be converted into an actual media, which is no longer virtual. This kind of approach seems to provide a new area in computational mechanics.

As a final comment, the present development seems to be suitable to the future computing environment. Since most of the software and hardware is designed based on the conventional computational methodologies, they are not necessarily relevant to the DIB computing. In other words, while the finite element method provides solution to the given problem, we always meet difficulties with the memory and storage capacity of computers. In fact, only linear problems are amenable to the present development of DIB-FE modeling. The nonlinear computation for a fully 3D structure is most often a formidable task even if we utilize a supercomputer. It is therefore natural to long for the novel computational method as well as the evolution of computer technologies and science.

\section{7}

\section{Conclusions}

We described why and how the digital image processing is used to make the microstructural FE model for the homogenization analysis. We also examined the DIB modeling from the technical and computational points of view. Here the "technical" means that the modeling techniques presented involve various kinds of manipulations for the digital images which are sampled, selected, thresholded or exported. Also, the homogenization analysis with the DIB modeling technique was found to feasible as RVE analysis. Moreover, the transition of volume fractional proportion and three-dimensional geometry of the microstructure was the virtually realized by manipulating the digital images. Although there were some uncertain factors in modeling, the microstructure seemed realistic. In summary, the DIB modeling with the asymptotic homogenization method is found to be the rigorous tool to study the complex micromechanical characteristics of composite materials.

At this end, it should be emphasized that we used only a few of digital image processing techniques. Considering the rate at which image science is presently developing, the authors strongly believe that digital images will change the conventional way of thinking. It seems to be time to draw our attention to applying digital images to computational mechanics field.

\section{References}

Bear, J. (1967): Dynamics of fluids in porous media. Dover Publications, Inc.

Berryman, J. G. (1985): Measurement of spatial correlation functions using image processing technique. J. Appl. Mech. 57, 2374-2384

Berryman, J. G.; Blair, S. C. (1987): Kozeny-Carman relations and image processing methods for estimating Darcy's constant. J. Appl. Phys. 62, 2221-2228

Carey, G. F.; Jiang, B. N. (1986): Element-by-element linear and non-linear solution schemes. Com. Appl. Num. Meth. 2, 145153

Dougherty, E. R. (1894): Introduction. In: Dougherty, E.R., (ed.): Digital image processing methods. Marcel Dekker Inc., pp. 1 
Duvaut, G. (1983): A new method of analysis of composite structure. Ninth European Rotor Craft Forum, No. 88 Ghosh, S.; Moorthy, S. (1995): Elastic-plastic analysis of arbitrary heterogeneous materials with the Voronoi cell finite element method. Comp. Meth. Appl. Mech. Eng. 121 (1-4), 373-409 Guedes, J. M.; Kikuchi, N. (1990): Preprocessing and postprocessing for materials based on the homogenization method with adaptive finite element methods. Comp. Meth. Appl. Mech. Eng. 83, 143-198

Haralick, R. M.; Shapiro, L. G. (1994): Glossary of computer vision terms. In: Dougherty, E.R. (ed.): Digital image processing methods. Marcel Dekker Inc. ed. Technomic Pub. Co.

Hollister, S. J.; Kikuchi, N. (1994): Homogenization theory and digital imaging: a basis for studying the mechanics and design principles of bone tissue. Biotechnol. Bioeng. 43 (7), 586596

Hollister, S. J.; Riemer, B. A. (1993): Digital image based finite element analysis for bone microstructure using conjugate gradi- ent and Gausian filter technique. Math. Meth. Med. Imag. II. 2035, 95-106

Hollister, S. J.; Brennan, J. M.; Kikuchi, N. (1994): Homogenization sampling procedure for calculating trabecular bone effective stiffness and tissue level stress. J. Biomech. 27 (4), 433-444 Léne, F.; Leguillon, D. (1982): Homogenized constitutive law for a partially cohesive composite material. Int. J. Solids Struct. 18 (5), 443-458

Sanchez-Palencia, E. (1980): Non homogeneous media and vibration theory. Lecture Notes in Physics 127, Springer-Verlag, Berlin

Terada, K.; Kikuchi, N. (1995): Nonlinear homogenization method for practical applications. In: Ghosh, S.; Ostoja-Starzewski, M. (eds.): Computational Methods in Micromechanics. AMSE AMD 212, 1-16

Terada, K.; Fonseca, J. O.; Kikuchi, N. (1996): Applications of the homogenization method to material design. In: International Symposium on Computer Modelling and Simulation for Material Design (CMSMD) '96, National Research Institute for Metals (to appear) 